\title{
Volume and surface photoemission from tungsten. II. Experiment
}

\section{Feuerbacher, B.; Egede Christensen, N.}

\section{Published in:}

Physical Review B

Link to article, DOI:

10.1103/PhysRevB.10.2373

Publication date:

1974

\section{Document Version}

Publisher's PDF, also known as Version of record

Link back to DTU Orbit

\section{Citation (APA):}

Feuerbacher, B., \& Egede Christensen, N. (1974). Volume and surface photoemission from tungsten. II. Experiment. Physical Review B, 10(6), 2373-2390. https://doi.org/10.1103/PhysRevB.10.2373

\section{General rights}

Copyright and moral rights for the publications made accessible in the public portal are retained by the authors and/or other copyright owners and it is a condition of accessing publications that users recognise and abide by the legal requirements associated with these rights.

- Users may download and print one copy of any publication from the public portal for the purpose of private study or research.

- You may not further distribute the material or use it for any profit-making activity or commercial gain

- You may freely distribute the URL identifying the publication in the public portal

If you believe that this document breaches copyright please contact us providing details, and we will remove access to the work immediately and investigate your claim 


\title{
Volume and surface photoemission from tungsten. II. Experiment
}

\author{
B. Feuerbacher \\ Surface Physics Division, European Space Research Organisation, Noordwijk, Holland \\ N. Egede Christensen \\ Physics Laboratory I, The Technical University of Denmark, 2800 Lyngby, Denmark
}

(Received 21 January 1974)

\begin{abstract}
Energy-distribution spectra of photoelectrons emitted normal to three single-crystal faces of tungsten have been measured for photon energies between 7.7 and $21.2 \mathrm{eV}$. The results are interpreted in terms of one-dimensional electronic properties along the symmetry lines in $k$ space that correspond to the emitting crystal faces. The emitted spectra may be considered to consist of three types of contributions. One part is due to electrons that, after excitation by direct interband transitions in the bulk, have left the crystal unscattered. This contribution may be described by the energy density of a one-dimensional joint density of states along a symmetry line in the Brillouin zone. Another part, evident for photon energies above $12 \mathrm{eV}$, is due to electrons that have suffered inelastic scattering processes after optical excitation. This part carries information on the density of conduction states along the symmetry line under observation. Finally, a third contribution to the emitted spectrum is assigned to surface emission. This part reflects the surface density of states and bears evidence of a narrowing of the $d$ bands near the surface. A theoretical surface density of states, derived from a simple model that scales the width of the $d$ bands by the square root of an effective number of neighbor atoms, is found to describe the energy distribution of the electrons emitted by the surface effect satisfactorily.
\end{abstract}

\section{INTRODUCTION}

The question whether external uv photoemission is primarily a bulk or a surface effect has been under discussion for a long time. Early theories ${ }^{1-3}$ have attributed photoemission solely to a surface effect. However, experimental results have shown that the crystal volume contributes significantly to the observed photoelectric emission. The conclusion was reached from measurements of the thickness dependence of the photoelectric yield of alkali metals, ${ }^{4-6}$ the correlation between photoelectric and optical properties, ${ }^{7,8}$ and the experimental observation of structure due to direct interband transitions in the energy-distribution spectra of photoemitted electrons. ${ }^{9-11}$ Therefore, experimental results have usually been discussed mainly in terms of bulk properties, ${ }^{12}$ assuming a working model of a three-step process including electron excitation by photon absorption, transport of the hot electron to the surface, and transmission through the surface. While the role of the surface effect alone has been discussed theoretically ${ }^{13-15}$ and experimentally, ${ }^{16,17}$ only very recently more rigorous theories have become available that treat both surface and volume effects in a unified manner, ${ }^{18-22}$ and as such question the validity of the experimentally very useful three-step concept. Rigorous calculations have been performed only for freeelectron-like metals ${ }^{18-21}$ and for a simple tightbinding model. ${ }^{23}$ For more complicated materials the three-step model appears to provide a good description of the experimental results, ${ }^{24,25}$ at least for noble metals, ${ }^{26-28}$ while difficulties arise in the case of transition metals with wider $d$ bands. 25,29

Unfortunately, it is not expected that the rigorous treatments of the photoelectric effect ${ }^{18-21}$ can be applied to complex materials, since the required knowledge of the initial- and final-state wave functions of the emitted electron is generally missing. In the analysis of the data presented here, a very simple heuristic approach has therefore been taken. From a phenomenological point of view structure in the photoelectron energy-distribution spectra is attributed to either surface or bulk excitation processes. The latter is treated following the conventional three-step model, while the surface emission is attributed to a one-step process in the spirit of the theories of Mahan, ${ }^{19}$ Schaich and Ashcroft, ${ }^{21,23}$ and Sutton. ${ }^{22}$ Using this approach, it is possible to relate the observed spectra to calculable quantities such as band structure and bulk or surface density of states.

The problem of experimental identification of bulk and surface contributions in the photoelectron spectra was tackled by narrowing the acceptance angle of the electron energy analyzer. After the early experiments of Gobeli, Allen, and Kane $\mathrm{e}^{30}$ showed that a large fraction of photoexcited electrons may leave the solid unscattered, a number of theoretical papers emphasized the importance of angular resolution in photoelectric measurements. ${ }^{19,23,31}$ Only very recently attempts were made to observe angular effects experimentally..$^{32-36}$ Serious difficulties were faced in these experiments, ranging from hardware problems due to complicated 
movements in ultra-high-vacuum systems to constraints imposed by the limited light intensities available in the vacuum-ultraviolet spectral region. The interpretation of the measured data turned out to be rather involved, ${ }^{33,34}$ unless the experimenter had chosen a particularly suited system. ${ }^{35}$ In the present work ${ }^{37}$ the measurements were taken at a fixed emission angle, namely, normal to a single crystal face, but the spectra were scanned for three different low-index faces. This approach is not only very simple from an experimental point of view (it is no more complicated than a "classical" large-acceptance-angle measurement), but it also allows an easier analysis of the results. The analysis assumes conservation of the momentum component parallel to the surface during electron emission. In that case the spectra should emphasize the electronic properties along the symmetry line in the Brillouin zone that corresponds to the crystal face under consideration. Band structure calculations are usually shown along symmetry lines, so a comparison between experimental results and theoretical predictions from band structure features is greatly facilitated.

The material chosen for the present investigation was tungsten. This choice was made mainly from an experimental point of view, since extensive studies of the surface properties are available, showing that three unreconstructed single crystal faces of tungsten can be prepared. Detailed information is found in the literature on the adsorption properties and cleaning procedures. Of special importance is the fact that the surfaces may be repeatedly cleaned under vacuum, thus permitting long observation times. A detailed relativistic band structure calculation for tungsten has been performed within the framework of the present study and is published in a separate paper. ${ }^{38}$ Properties relating to photoemission have been derived from this calculation and are compared to measured quantities. The $d$-band width of tungsten is about $11 \mathrm{eV}$, which is much larger than typical $d$ band widths of the noble metals. It is conjectured that with such a large $d$-band width, many-body effects due to heavy-hole localization will be minimal, so that spectral features other than those predicted by one-electron theory are avoided to a large extent.

The results of the directional photoemission measurements presented here allow the photoemitted electrons to be considered in three categories. A part of the electrons, excited by direct optical interband transitions in the bulk, may leave the crystal unscattered. It is those electrons that carry information on the bulk band structure along a specific symmetry line in $k$ space. These electrons may be described by a one-dimensional energy density of the joint density of states. For higher photon energies, another group of electrons becomes apparent, which have suffered inelastic scattering. These electrons closely resemble the one-dimensional density of bulk conduction states along the symmetry line under observation. Finally, a third group of emitted electrons is attributed to surface emission, carrying information on the surface density of initial states for vanishing parallel momentum. The former two groups appear to give good agreement with properties calculated from a bulk band structure. ${ }^{38}$ On the other hand, the experimental results give evidence for a narrowing of the $d$ bands at the surface. A simple model has been introduced ${ }^{38}$ that accounts for a narrowing of the $d$ bands at the surface as a consequence of the reduced $d$ - $d$ overlap resulting from the lowered number of neighbor atoms as compared to the bulk. Using this model the emission attributed to the surface effect may be described by a surface density of states in good agreement with the experimental spectra. Surface emission from a surface resonance is observed for the (100) face. Such a structure requires the calculation of the density of surface states for theoretical description which is not included in the present calculation of the surface density of states.

\section{METHOD OF INTERPRETATION}

The experimental photoelectron energy distribution spectra are interpreted here in a way that in some respects differs from the "classical" interpretation, which is based on the pioneering work of Berglund and Spicer. ${ }^{10,11}$ The differences originate from the experimental selectivity on the emitted electrons. The experimental setup is such that the excitation is as general as possible, i.e., unpolarized light at oblique incidence with a large cone of incidence angles. The main assumption in the interpretation of the present data is the conservation of the wave vector component parallel to the surface during electron emission, which is required by translational invariance along the surface. This assumption is the basis of all theoretical work on angular photoemission. ${ }^{19,21,31}$ In the present experiments, it may be justified by the fact that clean single-crystal surfaces are used which show sharp low-energy-electron-diffraction (LEED) patterns, indicating that specularity is fulfilled at least for high electron energies. In the experimental configuration used, where only normally emitted electrons are measured, conservation of parallel wave vector means small or vanishing parallel wave vector both inside and outside the crystal.

A second assumption important for the interpretation of the results is that elastic scattering is negligible. This means that the "secondary cones"19 due to electrons that have taken up one or more 
additional reciprocal lattice vectors after photoexcitation (umklapp process) are weak. It is obvious that such secondary cones must exist, since a Bloch wave inside the material cannot be matched to a single plane wave outside. However, it is assumed that those contributions are weak in intensity. The effects of phonon scattering, characterized by a negligible loss in energy but large momentum transfer, are also included in the term elastic scattering. The justification for neglecting these effects is based on the experimental observation that regions of high density of states along a certain symmetry line do not contribute significantly to photoemission along another symmetry line. Examples for this observation are discussed later.

The assumptions of specularity and negligible elastic scattering lead to the present interpretation of the experiments. Spectra observed for electrons emitted normal to a crystal face are related to one-dimensional electronic properties along the symmetry line that corresponds to this face, as far as volume effects are concerned. For surface effects, they are related to the associated surface density of states.

\section{A. Direct transitions}

Electrons emitted after direct interband transitions are treated following the three-step model, assuming optical dipole excitation in the bulk, followed by transport and emission. The experiment allows a clear distinction between unscattered and inelastically scattered electrons. Attention is focussed here onto the unscattered part, which should carry information on the bulk band structure along a certain symmetry line. It may be pointed out that even in the more rigorous treatment of photoemission the bulk contribution still bears resemblance of the joint density of states, ${ }^{23}$ at least as far as the position of structure in the spectra is concerned. Observed structure is therefore compared to theoretical calculations of the onedimensional energy density of the joint density of states. ${ }^{38}$

The experimental identification of structure due to direct transitions is made as usual by observation of the movement of the initial state energy with varying photon energy. This assignment is generally not quite unique, especially if the initial band is flat and the final band steep. However, the limitation to a single symmetry line cuts down the number of expected direct transitions drastically. Therefore, with the aid of a calculated band-structure diagram, the assignment of structure to direct transitions is no real problem. Note that direct transitions observed in the way described here require conservation of the normal wave-vector component during excitation, so they can be regarded as a true bulk process.

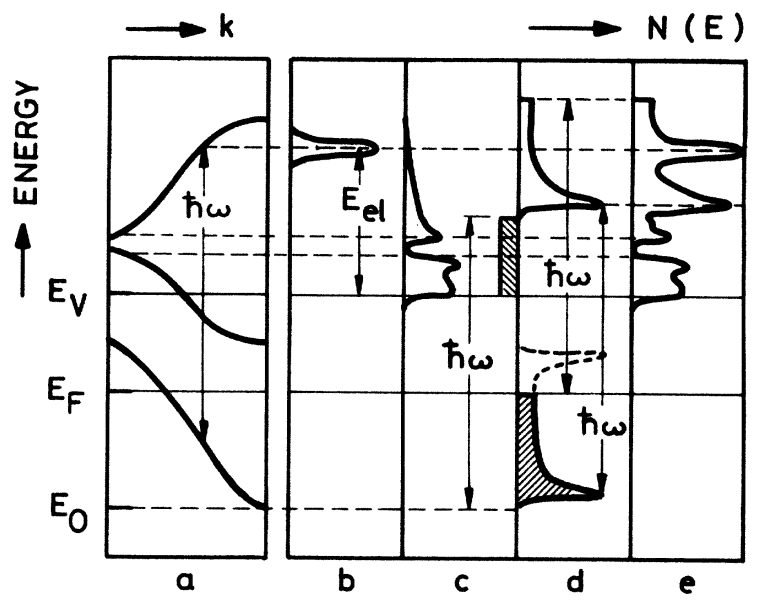

FIG. 1. Part of a hypothetical band structure (a) used to demonstrate schematically the various contributions to the observed photoemission spectra; (b) direct-transition contribution; (c) inelastically scattered electrons reflecting the final density of states; (d) surface emission resembling the surface density of states; (e) total spectrum.

A sketch of the mechanism involved in the emission due to direct optical transitions is shown in Fig. 1. Figure 1(a) shows a slice of a hypothetical band structure along a symmetry line. For the photon energy $\hbar \omega$, only one direct interband transition is possible. This is indicated by the vertical arrow. In the absence of inelastic scattering the transition will give rise to a single peak of photoelectrons as shown in Fig. 1(b), measured at an energy $E_{\mathrm{el}}$ above the vacuum level. If the photon energy is increased by an amount $\Delta \hbar \omega$, the energy $E_{\text {e1 }}$ will increase by a smaller amount. Since photoemission spectra are usually referred to initial-state energy, this manifests itself in the above-mentioned movement of the initial-state energy characteristic for direct transitions.

\section{B. Inelastically scattered electrons}

Electrons excited by direct interband transitions in the bulk may suffer inelastic scattering events before escaping the surface. These events may be described by electron-electron scattering (pair production), electron-hole scattering (Auger process), or plasmon excitation. The latter is expected to be relatively unimportant in a material like tungsten, where the plasmon resonance is highly damped. The former two processes have been discussed by $\mathrm{Kane}^{39}$ and by Nilsson. ${ }^{40}$ It is found that electron-pair production is the dominant loss process for the energies involved in ultraviolet photoelectron spectroscopy.

The mathematical treatment of the electron-electron scattering process shows that the final density 
of states may be factored out in the expression for the energy distribution of the emitted electrons. ${ }^{39-41}$ In the present selective experiment this means that the scattered electrons carry information on the onedimensional conduction-band density of final states along the symmetry line corresponding to the emitting surface. An illustration of this is given in Fig. 1. Figure 1(c) shows the contribution of scattered electrons. They reflect a gap in the band structure shown Fig. 1(a). It should be borne in mind that for scattered electrons the directional photcemission is only selective on the final state. So, in the example of Fig. 1, the contribution in section $c$ is not only composed of the electrons scattered off the peak due to direct transitions for the same photon energy [Fig. 1(b)]. All possible interband transitions for $\Delta E=\hbar \omega$ in the entire Brillouin zone may contribute to the inelastic spectrum, as long as their final state after scattering is found on the symmetry line under observation.

Experimentally, the identification of inelastically scattered electrons is unambiguous only for high enough photon energies. That is if the exciting energy is larger than the difference between the bottom of the valence band $E_{0}$ and the vacuum level. All electrons emitted with energies between the vacuum level and $E_{0}+\hbar \omega$ [shaded area in Fig. 1(c)] must have undergone inelastic scattering events before escaping.

\section{Surface emission}

As shown above, the probability of detecting direct interband transitions in directional photoemission is quite limited. There is, however, some photoemission structure found in regions where it cannot be attributed to either direct transitions or inelastic scattering. For example, no final states are available along the [110] symmetry line of tungsten in the range up to about $5 \mathrm{eV}$ above the vacuum level. Yet there is considerable photoelectric structure found in this range. A lack of final states means that there are no Bloch states available to transport electrons from the bulk to the surface with the appropriate $k$ vector for normal emission. It is therefore assumed that photoemission in such a range could be attributed to a surface effect.

Surface photoemission is considered as a onestep process ${ }^{13,19,21}$ in which a transition takes place from an initial state localized near the surface to a free-electron-like state outside the crystal. It has been shown ${ }^{22,23}$ that photoelectron spectra due to such a process should resemble the density of initial states at the surface. As a consequence, the position of structure in the energy distribution curves should be independent of photon energy, if plotted as a function of initial state energy. On the other hand, if, as will be assumed, the transitions matrix elements vary slowly with excitation energy, no strong modulation of the intensity of structure due to surface emission is expected as the photon energy is varied. These properties will serve as criteria for experimental identification of photoelectrons emitted from the surface.

An illustration of this type of emission is given in Fig. 1(d). The cross-hatched area indicates those occupied states at the surface having zero components of parallel wave vector. The density of these states is not necessarily the same as in the bulk. (It will be shown later, that for tungsten the bands appear to be narrower at the surface than in the bulk.) This structure is projected upwards by an amount equal to the photon energy $\hbar \omega$, with possible but as yet unknown distortions. Although this type of photoemission process in many respects appears similar to the "non-direct" process proposed by Spicer, $42,43,10,11$ there is, however, a basic difference. Nondirect transitions are a volume effect consisting in transitions from an initial to a final Bloch state in which wavenumber is not conserved but energy is. In the present experiment this would mean that electrons with initial states in many areas in $k$ space could contribute to a final band state that is detected by the external selection mechanism. The experimental evidence shows that such contributions must be weak, i.e., bulk optical transitions appear to have both initial and final states at the symmetry line selected by the experiment.

\section{EXPERIMENTAL}

A schematic layout of the experimental setup is shown in Fig. 2. Light from a monochromator is collected by a spherical gold-coated mirror and focused onto the sample within a spot size of about $1 \times 0.5 \mathrm{~mm}$. The angle of incidence ranges from nearly grazing to about $30^{\circ}$. A uniform field is obtained around the sample by a hemicylindrical screen with grid openings for the light. The radius of the screen is $7 \mathrm{~mm}$. This small size makes the instrument fairly insensitive to ambient magnetic fields. A small accelerating potential of about $1 \mathrm{~V}$ was placed between sample and screen. A hole of $1.5 \mathrm{~mm}$ in diameter in the center of the screen is coincident with the entrance slit of the electron energy spectrometer. This arrangement allowed only electrons emitted within a cone of $12^{\circ}$ full opening centered normal to the sample to enter the analyzer. Alignment of the sample normal was achieved by means of a laser beam used in autocollimation.

The samples were cut from single-crystal tungsten of $99.95 \%$ purity in the form of bars with dimensions $20 \times 2 \times 0.5 \mathrm{~mm}$. The polished surfaces of the crystals were parallel to the (100), (110), 


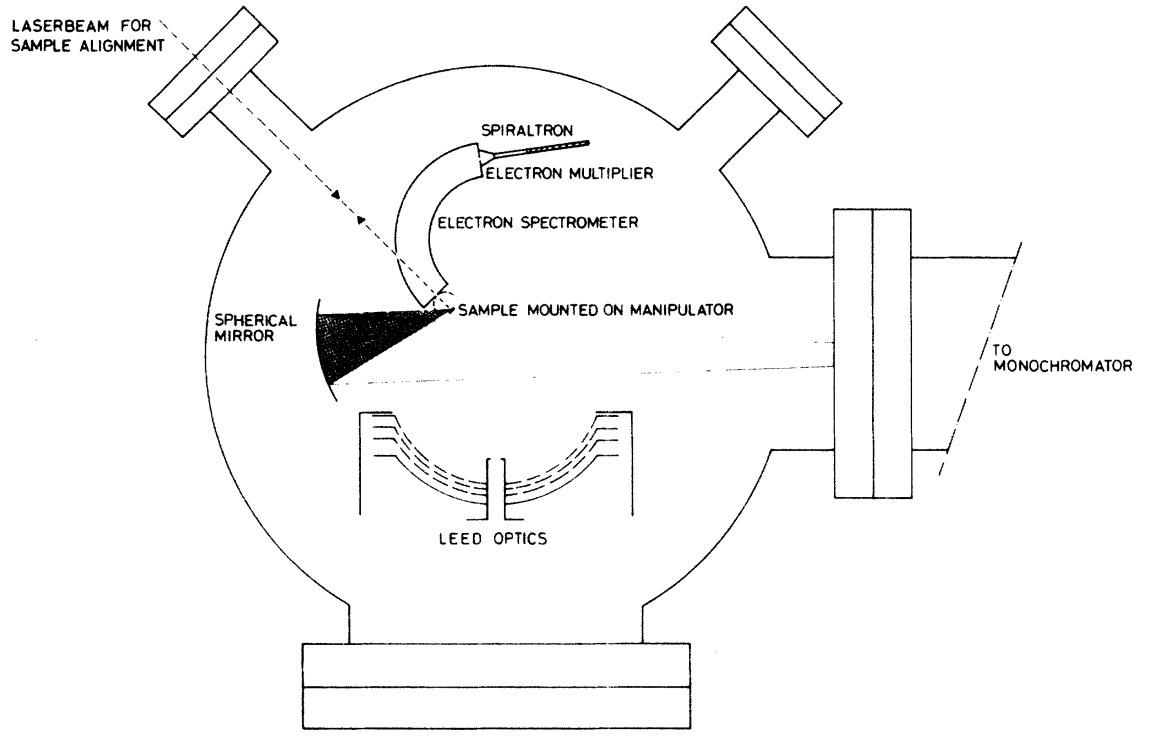

FIG. 2. Schematic layout of the experimental chamber (from Ref. 51).

and (111) planes to within $3^{\circ}$. All crystals were precleaned in a separate LEED-Auger System by successive heating and oxygen etching, until Auger scans showed that no carbon had diffused from the bulk to the surface during the heating cycle. Additional oxygen etching took place in the experimental chamber. Oxide on the surface was removed by flashing to $2300^{\circ} \mathrm{C}$ for one minute. Prior to each experiment the sample was transferred from the experimental position described above to a LEED optics in order to check for surface structure and cleanliness. The crystals showed sharp diffraction patterns and no contamination could be detected by Auger spectroscopy. For each crystal face, measurements have been performed on at least two independently prepared surfaces.

Energy analysis of the photoelectrons was performed in a $127^{\circ}$ cylindrical electrostatic analyzer. With a mean radius of curvature of $40 \mathrm{~mm}$, a theoretical resolution of about $2 \%$ is expected. Spectra were scanned by ramping the potential between the cylinder plates. This scanning mode keeps the electrostatic environment near the sample constant, but some distortion is introduced in the measured spectra by the variation of transmission and resolution with electron energy. The performance of the analyzer is shown in Fig. 3. Transmission was measured by accelerating photoelectrons emitted at constant photon energy by a field applied between sample and analyzer entrance aperture. Due to the nonspherical geometry, focussing effects prevented reliable results for accelerating fields above $7 \mathrm{~V}$. The resolution was determined by the same method, using the sharp high-energy cutoff edge of "dirty" tungsten as a reference. Except for very low energies, the resolution was found to be $2.3 \%$, which is close to the theoretical value of $2 \%$ derived from the analyzer geometry.

A channeltron electron multiplier was placed behind the spectrometer exit slit to detect the transmitted electrons. Counts were stored in a 500-channel multiscaler, whose channel address controlled directly the plate potential of the analyzer. The spectra were scanned repeatedly until a signal-to-noise ratio of about $1 \%$ was obtained. From the multiscaler, the data was transferred to a computer in digital form. Here the curves were corrected for the variation in analyzer transmission, approximated by the straight line shown in

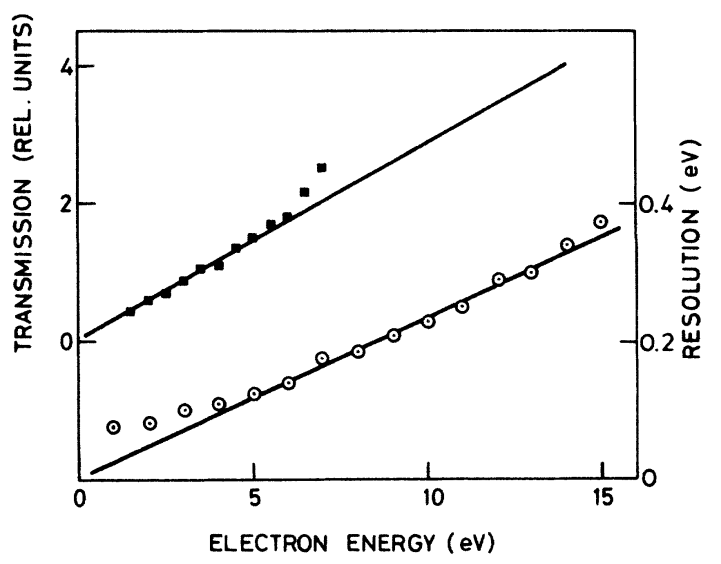

FIG. 3. Transmission and resolution of the electrostatic deflection analyser as a function of photon energy. The scale for the transmission (left-hand side) is shifted upwards. 


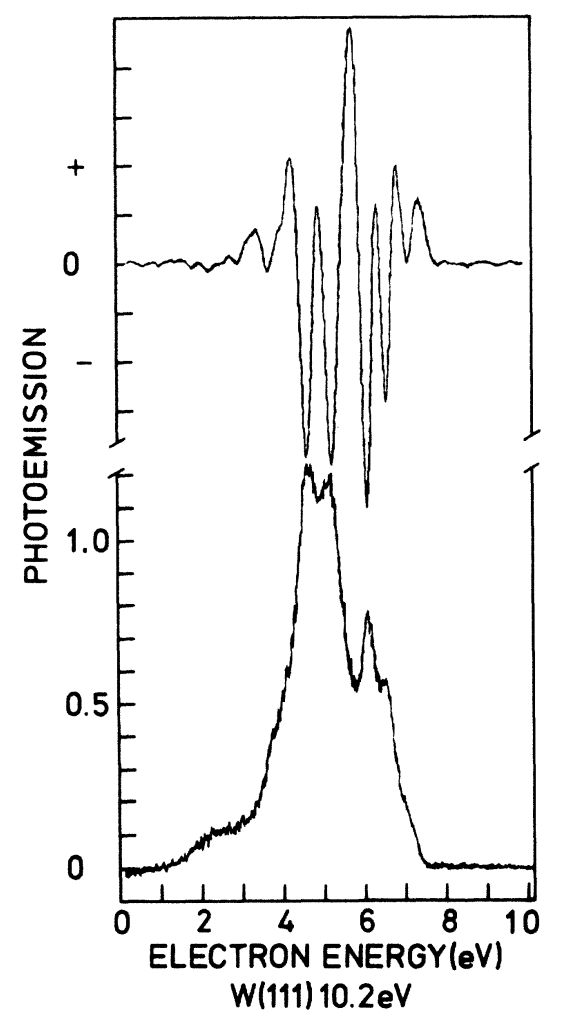

FIG. 4. Example for an experimental photoelectron spectrum (bottom curve) taken normal to the (111) face of tungsten at 10.2-eV photon energy. The top curve shows the second derivation of the same spectrum. A derivative interval of $0.4 \mathrm{eV}$ was used.

Fig. 3. No correction on the variation of resolution with energy was applied. A typical energy distribution spectrum as plotted by the computer is shown in the bottom curve of Fig. 4. The upper curve gives the second derivative calculated using a differentiation interval of $0.4 \mathrm{eV}$. The apparent enhancement of structure in the second derivative allows the position of structure to be obtained more accurately than from the direct spectrum. In addition, computer differentiation offers a considerable improvement in signal-to-noise ratio as compared to direct electronic differentiation of the actual emission current. No smoothing was applied to the curves shown in Fig. 4.

During all measurements at photon energies below $11.7 \mathrm{eV}$ the experimental chamber was closed by a lithium-fluoride window. The pressure during the measurements was in the $10^{-11}$ Torr range. Scan times were such that no more than 0.05 of a monolayer of adsorbants could be accumulated, assuming a unity sticking coefficient. A clean surface was restored whenever necessary by resistive heating to about $2500^{\circ} \mathrm{C}$ for a few seconds. For higher photon energies, the lithium fluoride window had to be removed. A three stage differential pumping system between the light source and the monochromator allowed a working pressure of $1 \times 10^{-9}$ Torr in the experimental chamber. Scan times of $10 \mathrm{sec}$ per spectrum and heat cleaning of the crystal between successive scans ensured measurements on clean surfaces even under these conditions.

\section{RESULTS}

\section{A. Emission normal to the (100) face}

The results of directional photoemission measurements will be discussed mainly in terms of one-dimensional features along symmetry lines in the Brillouin zone, as described in Sec. II. For emission normal to the (100) face the corresponding symmetry line is the $\Gamma H$ line. In Fig. 5 some calculated electronic properties are shown along this line. The left-hand part gives a section of the band structure as obtained by the relativistic augmented-plane-wave (APW) method. ${ }^{38}$ The righthand part shows the one-dimensional density of states for the same symmetry line. Details of the calculations leading to Fig. 5 are discussed elsewhere. ${ }^{38}$

A set of photoelectron energy distribution curves as measured normal to the (100) face of tungsten is shown in Fig. 6. The curves have been taken

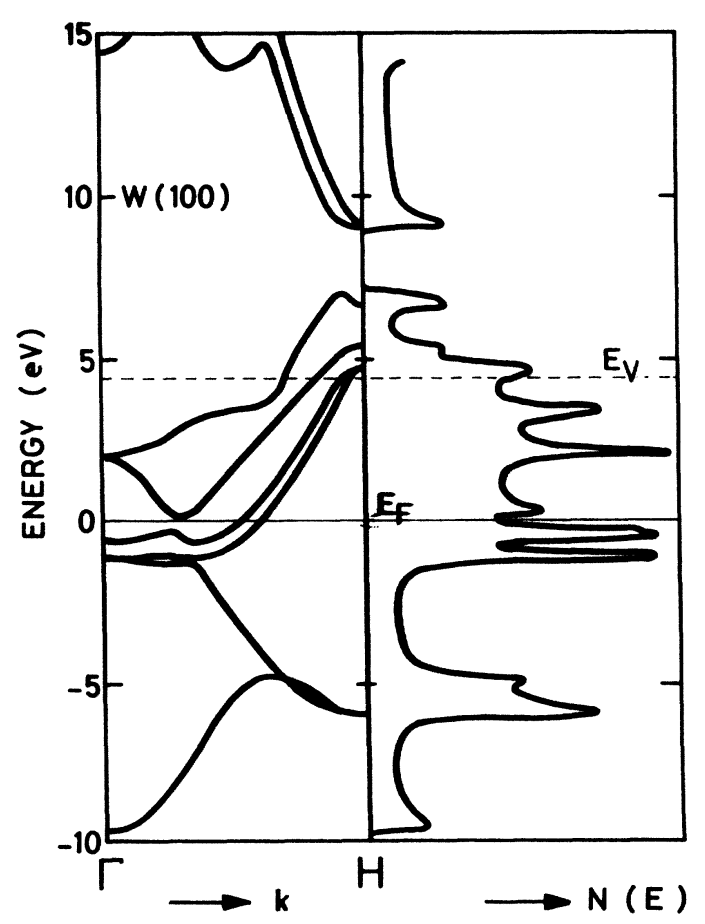

FIG. 5. Calculated band structure of tungsten along the $\Gamma \mathrm{H}$ symmetry line (left-hand side) and one-dimensional density of states (right-hand side). 


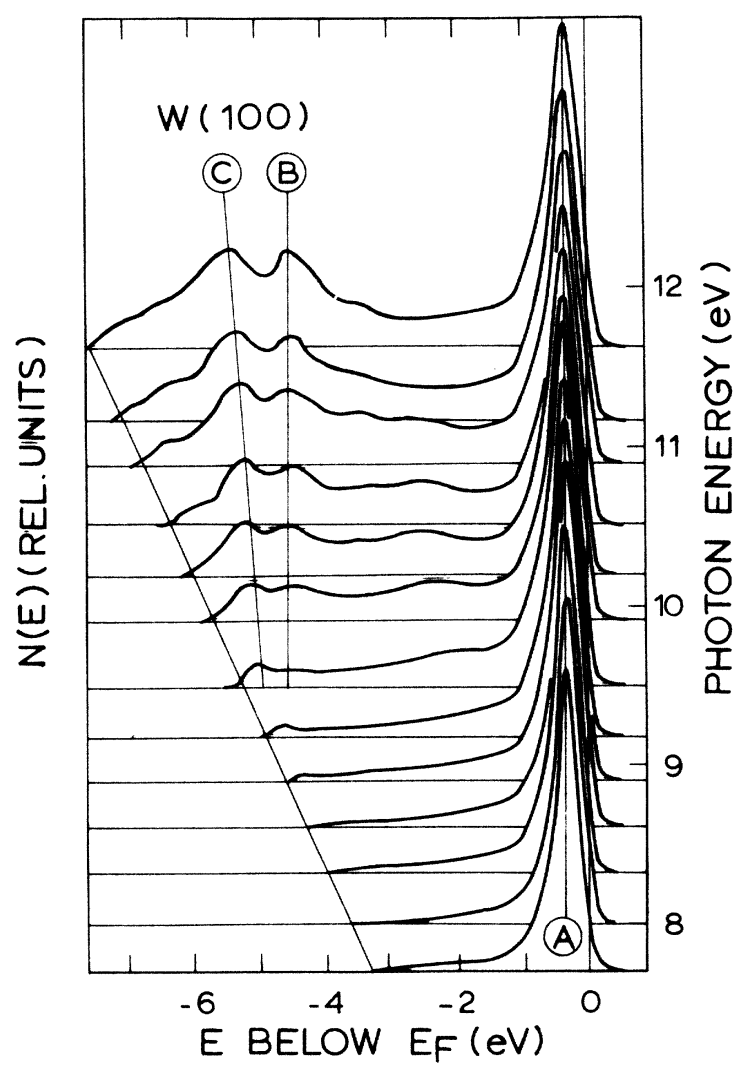

FIG. 6. Experimental energy-distribution spectra of photoelectrons emitted normal to the $(100)$ face of tungsten for photon energies between 7.7 and $11.7 \mathrm{eV}$. Peak $A$ is assigned to a surface resonance, structures $B$ and $C$ to surface emission and bulk direct transitions, respectively.

at different photon energies, and the baselines are shifted vertically by an amount proportional to the excitation energy difference, such that the photon energy may be read from the right-hand scale. The bottom scale refers to initial-state energy relative to the Fermi level, i. e., the photon energy has been subtracted from the measured kinetic electron energy and the work function added:

$$
E=E_{\mathrm{kin}}+E_{\mathrm{w} \mathbf{F}}-\hbar \omega .
$$

An electron found at $E=0$ therefore has been emitted right from the Fermi surface.

An attempt was made to normalize the various curves relative to each other in such a way that the area under each curve is proportional to the number of photoelectrons emitted into the analyzer acceptance cone at the respective photon energy. However, in the present experimental arrangement it was not possible to measure the electron current restricted normal to the surface. Instead of this the total yield from the (100) surface has been measured as a function of photon energy, and the measured spectra were scaled using these values.

The most pronounced structure in Fig. 6 is the strong leading peak labeled A. This peak appears to be independent of photon energy both as far as size and position are concerned. A similar structure has been observed in a photoemission experiment by Waclawski and Plummer ${ }^{44}$ and in the energy distribution of electrons field emitted from the (100) face of tungsten as measured by Plummer and Gadzuk. ${ }^{45}$ The fact that the structure is also observed in field emission normal to (100) face leads to the conclusion that it should be due to an initial density-of-states effect, in agreement with the observation that it is independent of photon energy. A glance at the one-dimensional density of states (Fig. 5) shows that two peaks of nearly the same size are calculated just below the Fermi level. These are separated by $0.7 \mathrm{eV}$ and therefore should be clearly resolved by the experiment if they were responsible for the structure. In the literature structure $A$ has been assigned to emission from a surface resonanc $e^{44-46}$ in a band gap arising from spin-orbit splitting, similar to the surface states in the $s-d$ gap predicted by Forstmann, Heine, and Pendry. ${ }^{47,48}$ Gadzuk $^{49}$ has performed calculations on this basis and finds good agreement with field-emission measurements. ${ }^{45}$ However, a surface state has never experimentally been observed in a $s-d$ band gap, ${ }^{\mathbf{5 0}}$ and no indication for such surface states is found in other gaps in the tungsten band structure.

While the theoretical basis for the assignment of structure $A$ to a surface resonance appears to be somewhat questionable, there is some experimental evidence in favor of the assignment to surface emission. The band structure calculations show that direct optical transitions in the bulk do not contribute to this structure. This is in line with the fact that no final state modulation is observed (the structure is independent of photon energy) and that the peak is also found in field emission experiments. Striking evidence for its origin at the surface is obtained from gas adsorption measurements. Note that a very similar leading peak is observed in the photoelectron spectra taken normal to the (110) face (see Fig. 13), which, as will be shown below, can be assigned partly to emission due to direct optical excitations in the bulk. In Fig. 7 the effect of gas adsorption is compared for those two structures. Here the relative height of the leading peak for the (100) and (110) emission is plotted as a function of exposure to hydrogen. ${ }^{51}$ It is seen that the (110) leading peak decreases slowly with exposure, while the structure assigned to a surface resonance virtually disappears for exposures corresponding to a coverage of a fraction of a monolayer.

In the photoelectron spectra shown in Fig. 6 two 


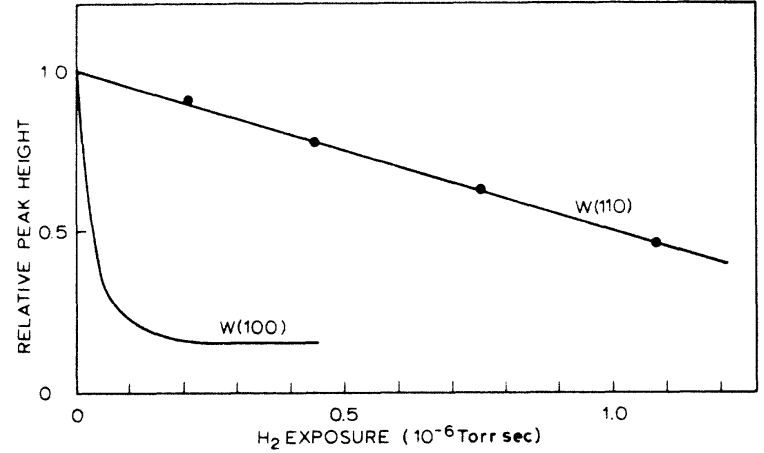

FIG. 7. Relative peak height of the leading peak in the (100) spectra (peak $A$ in Fig. 6) and the (110) spectra (peak $A$ in Fig. 12) as a function of hydrogen exposure at 10.2-eV photon energy.

other prominent features are observed, labeled $B$ and $C$. Structure $B$ is found to originate from a constant initial state at $-4.5 \mathrm{eV}$, while the initialstate energy of structure $C$ decreases with increasing photon energy in a way characteristic for emission due to direct optical transitions. The band structure in Fig. 5 shows that direct optical transitions are possible from the two lowest bands, nearly degenerate close to $H$ to final states above the vacuum level, for photon energies above 9.5 eV. Such transitions would originate from initialstate energies decreasing with increasing photon energy, in agreement with the behavior of peak C. It is shown in Fig. 8 that this agreement is not only qualitative but also quantitative. The position of structure arising from direct transitions along the $\Gamma H$ symmetry line has been derived from the calculated band structure, using the energy density of the joint density of states to describe photoemission due to direct optical transitions. ${ }^{38}$ The result is plotted in Fig. 8 for photon energies between 10 and $13 \mathrm{eV}$ (solid line) and for $16.8 \mathrm{eV}$ (cross). In the same figure the experimental peak positions are shown as circles. The correlation between experimental and theoretical results is very good with deviations of no more than $0.1 \mathrm{eV}$ over the whole photon energy range. On the basis of this evidence the process leading to structure $\boldsymbol{C}$ is assigned to photoemission following direct optical excitations in the bulk.

Following the ideas outlined in Sec. II C, the stationary structure $B$ in Fig. 6 is attributed to a surface photoelectric effect (the increase in peak height with increasing photon energy is probably due to the threshold function acting on the low-energy part of the photoemitted electrons). Another possible explanation for structure $B$ could be nondirect transitions ${ }^{42}$ from the high density-of-states point associated with the maximum in the lowest band in Fig. 5. However, this assignment is refuted on the basis of the following agruments. If nondirect processes were important in tungsten, transitions from initial states all over the Brillouin zone to final states along the [100] symmetry line should contribute to the observed spectra. But all energy distribution curves in Fig. 6 show little emission from states in the range 1.5-4 eV below the Fermi level. Now the calculated total density-of-states curve for tungsten ${ }^{38}$ shows regions of high densities of states in other parts of the Brillouin zone in the above energy range. Thus, the observation that contributions of such regions are small leads to the conclusion that nondirect emission processes are unimportant in tungsten.

Other evidence against the assignment of structure $B$ to nondirect transitions is deduced from considerations involving final-state modulation. The density-of-states curve along the [100] direction shown in Fig. 5 exhibits a band gap extending from 7 to $9 \mathrm{eV}$ above the Fermi level. If the structure at $-4.5 \mathrm{eV}$ is due to nondirect transitions, it should disappear for excitation energies between 12 and $13 \mathrm{eV}$, when the final state energy is in the band gap. In Fig. 9 photoelectron energy distribution spectra in the corresponding photon energy range are presented. ${ }^{52}$ It is seen that the -4.5-eV structure does not vanish at all for these excitation energies, excluding nondirect emission as a possible explanation for peak $B$.

It still remains to be shown that the energy gap used to discard the assignment of peak $B$ to nondirect transitions actually is found at the position where it is predicted by the band-structure calculation. This can be done using the inelastically scattered electrons, which are expected to carry information on the final density of states (see Sec.

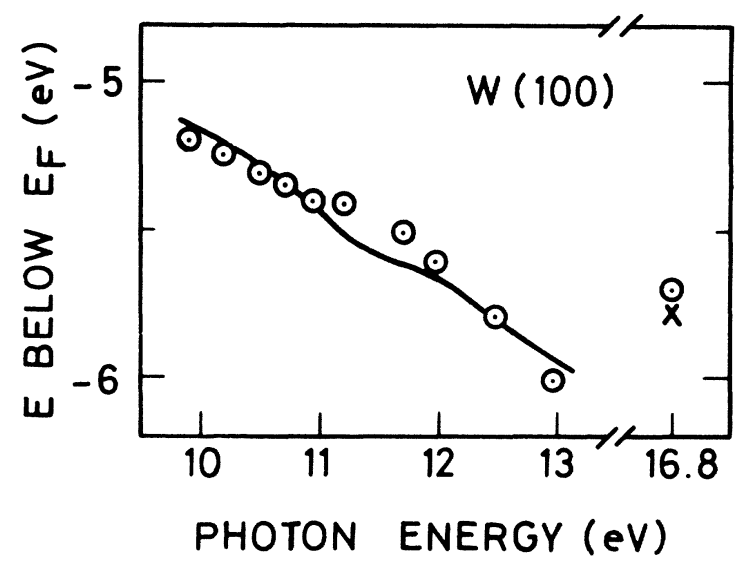

FIG. 8. Movement of initial-staie energy of structure assigned to emission following direct optical excitation (peak $C$ in Fig. 6) as a function of photon energy. Solid line and $\times$, calculated; $\odot$, measured peak position. 


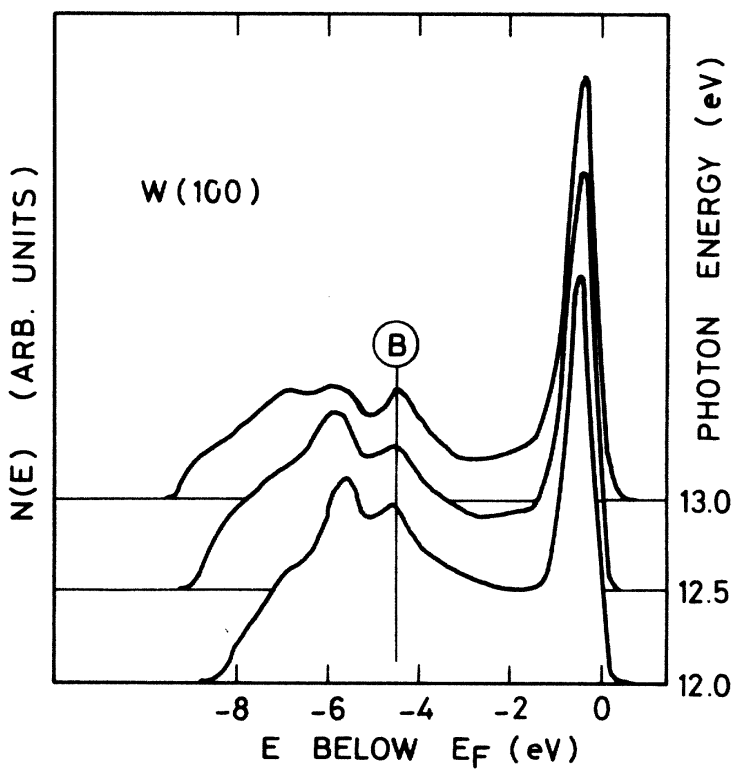

FIG. 9. Energy distribution spectra of photoelectrons emitted normal to the (100) face of tungsten for photon energies between 12 and $13 \mathrm{eV}$.

II B). While the photoelectron spectra for excitation energies up to $13 \mathrm{eV}$ show little inelastic electrons, those become much more apparent for higher photon energies. In Fig. 10 spectra are shown for 16. 8- and 21.2-eV photon excitation. The two upper curves are placed relative to each other such that the horizontal scale refers to final state energy relative to the Fermi level, i. e., the work function has been added to the measured kinetic energy of the emitted electrons. The 16.8-eV curve shows a low-energy hump of scattered electrons which is comparable in size to the unscattered structure on the high-energy side, while for $21.2 \mathrm{eV}$ the low-energy electrons are dominant. ${ }^{52}$ This is consistent with the expected decrease of the mean free path of hot electrons with increasing energy in this range. ${ }^{53,25}$

Two peaks marked $G$ and $F$ are observed at the same final-state energy in both curves. The dashed line marked DOS is a part of the one-dimensional density-of-states curve from Fig. 5. The minimum between structures $G$ and $F$ appears to correlate well with the calculated band gap in the density of states, and the two peaks may be attributed to the two maxima associated with this gap. It appears that the calculated band structure gives good agreement with the measured quantities up to at least $10 \mathrm{eV}$ above the Fermi level.

The lower curve in Fig. 10 is the same as the top curve, but shifted horizontally such that the bottom scale refers to initial state energy. The structures labeled $A, B$, and $C$ are seen to coin- cide in both the 16.8-eV and 21.2-eV spectra, while $D$ and $E$ do not. The dashed line shows a calculation of the photoemeission contribution due to direct interband transitions for $16.8-\mathrm{eV}$ excitation energy. Details of this calculation, which includes only electrons emitted into $a \pm 6^{\circ}$ cone normal to the surface, are discussed in a separate paper. ${ }^{38}$ Good agreement is found for the structures $A, B$, and $D$ in the $16.8-\mathrm{eV}$ curve, but peak $C$, which appears also in the $21.2-\mathrm{eV}$ curve, is not described by this calculation. This is not surprising since peak $C$, found at $-4.5 \mathrm{eV}$, is the same structure observed in all photoemission spectra normal to the (100) face (labeled $B$ in Figs. 6 and 9) and assigned to surface photoemission, which of course is not included in the direct-transition model leading to the dashed line in Fig. 10.

An attempt was made to describe the structure due to surface emission in a more quantitative way. This was based on the work of Schaich and Ashcroft $^{23}$ who showed that structure in the surface photoemission is roughly related to structure in the surface density of states. Extending the bulk density of states to the surface did not yield satisfactory results, since, as shown below, the width of the $d$-bands appears to be narrower at the surface than in the bulk. Haydock et al. ${ }^{\mathbf{5 4}}$ have noted that the width of the $d$-bands at the surface should vary approximately proportional to

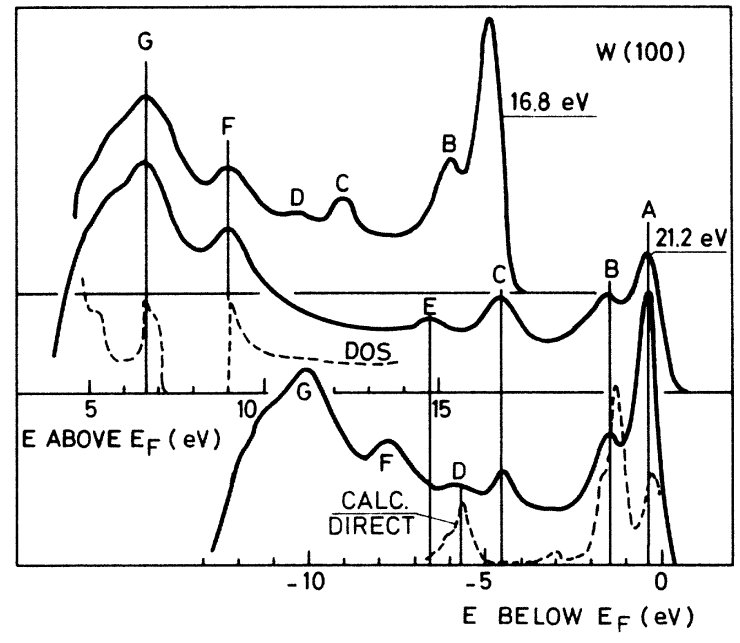

FIG. 10. Energy-distribution curves of photoelectrons emitted normal to the (100) face of tungsten for 16.8and 21.2-eV photon energies. Top and bottom curve are identical $(16.8 \mathrm{eV})$ but shifted horizontally such that the upper horizontal scale refers to final-state energy and the bottom scale refers to initial-state energy. The dashed lines give calculated curves for the density of states (DOS) or the contribution due to direct optical transitions (Calc. Direct) at 16.8-eV excitation energy. 


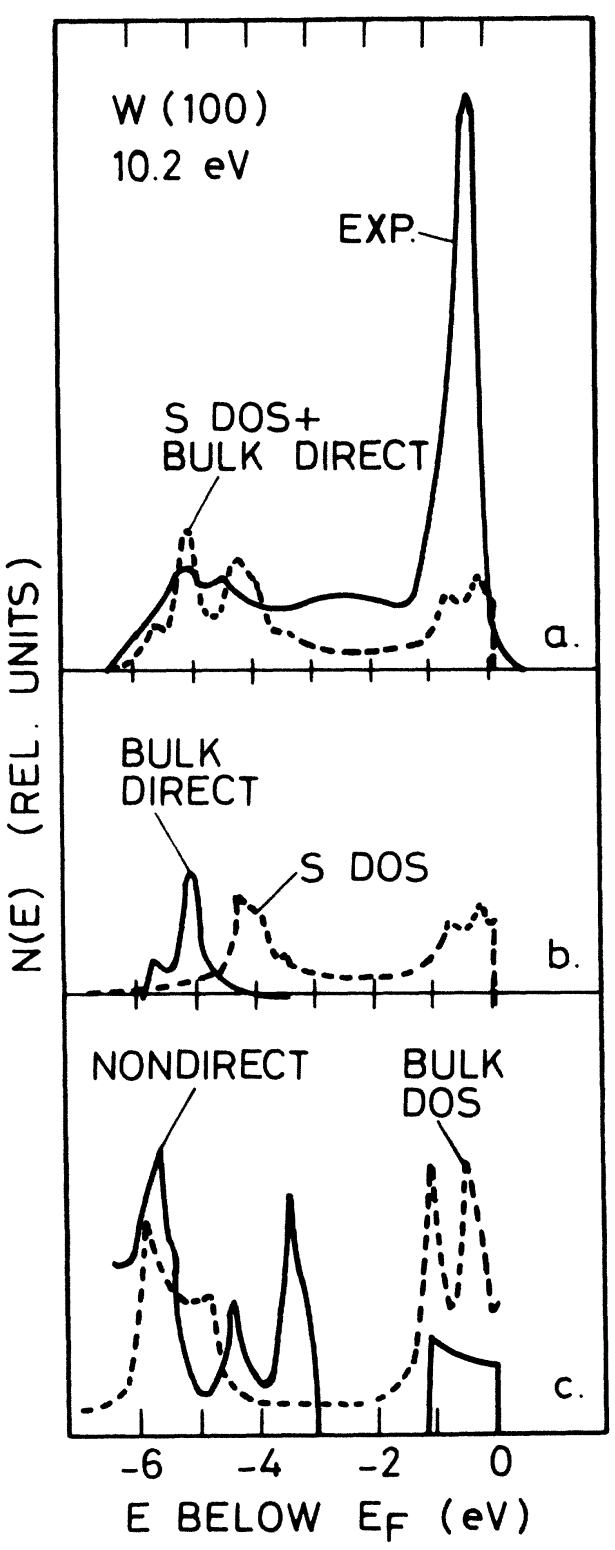

FIG. 11. Photoemission normal to the $(100)$ face of tungsten at $10.2-\mathrm{eV}$ photon energy compared to various calculated models. (a) Solid curve, experimental energy distribution spectrum; dashed curve, sum of bulk direct transition and surface emission contributions. (b) Calculated spectra assuming bulk direct transitions (solid curve) or surface emission derived from a surface density of states (dashed curve SDOS). (c) Bulk density of states (dashed curve) and calculated spectrum assuming non-direct transitions (solid curve).

the square root of the number of nearest neighbor atoms. This approximation was used in a crude reduced-overlap-calculation model, set up to account for the narrowing of the $d$-bands at the surface. This model is described in detail elsewhere. ${ }^{38}$
In Fig. 11, a comparison is made between the measured photoelectron energy distribution spectrum normal to the (100) face for $10.2-\mathrm{eV}$ photon energy and various calculated spectra. The solid curve in part a shows the measured spectrum. In Fig. 11(b), the solid line shows the calculated contribution due to bulk direct transitions in $a \pm 6^{\circ}$ cone. The peak at $-5.2 \mathrm{eV}$ is in good agreement with the measured structure assigned to bulk direct transitions (peak $C$ in Fig. 6). A calculated surface density of states using the above model, ${ }^{38}$ shown by a dashed line in Fig. 11(b), gives reasonable agreement with the peak assigned to surface emission (peak $B$ in Fig. 6). Figure 11(c) demonstrates that this structure is not well described by the bulk density of states (dashed curve). A calculation assuming nondirect excitation in the bulk is presented as a solid curve in Fig. 11(c). While the structure at $-4.5 \mathrm{eV}$ could be attributed for by such a process, a much stronger peak is predicted at $-3.5 \mathrm{eV}$ for which there is no evidence in the experimental spectra.

The dashed line in Fig. 11(a) represents the sum of the contributions from the direct model and the surface emission model, as shown in Fig. 11(b) separately. The two structures on the lowenergy side are reasonably reproduced by this

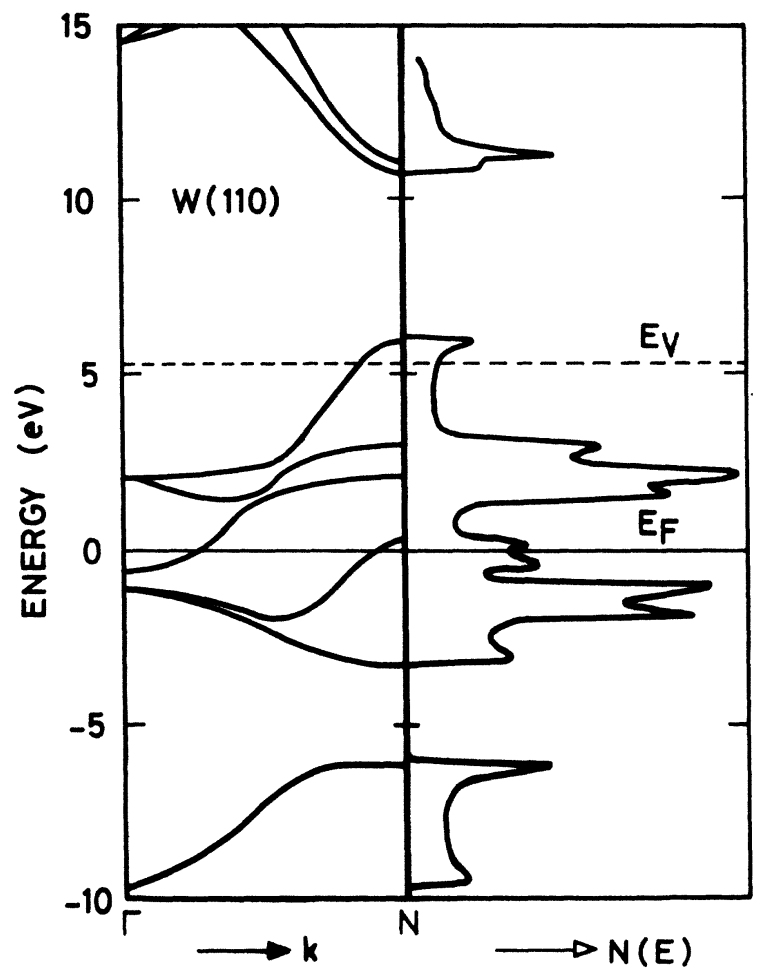

FIG. 12. Calculated band structure of tungsten along the $\Gamma N$ symmetry line (left-hand side) and one-dimensional density of states (right-hand side). 


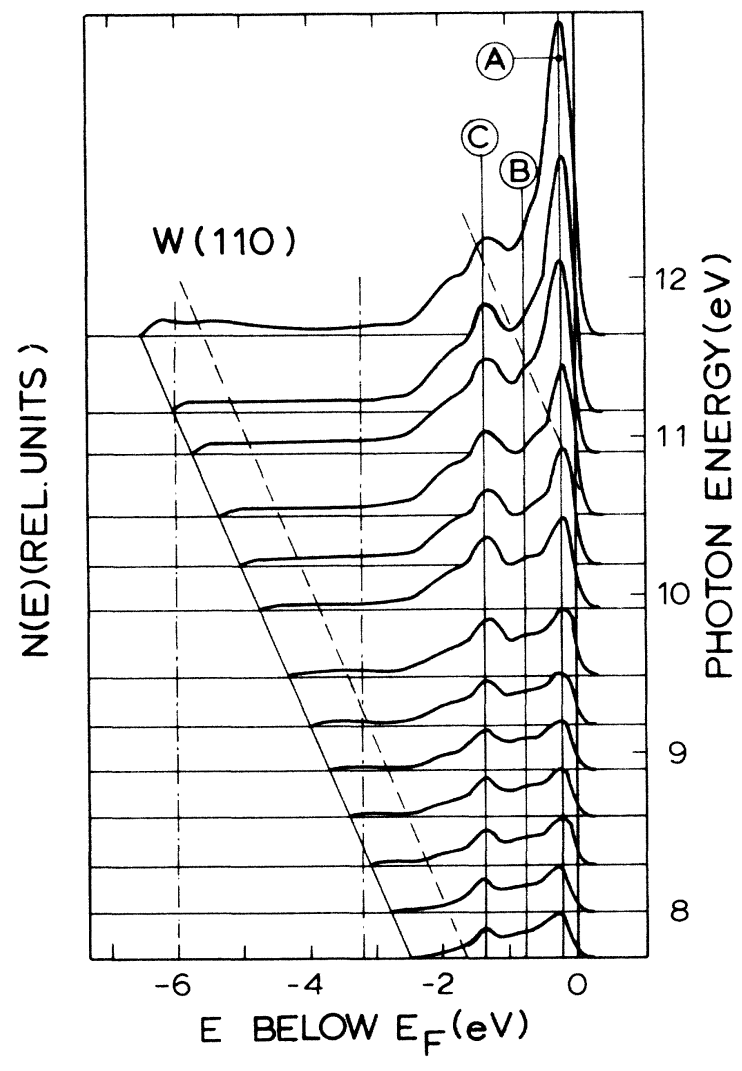

FIG. 13. Experimental energy-distribution spectra of photoelectrons emitted normal to the (110) face of tungsten for photon energies between 7.7 and $11.7 \mathrm{eV}$. The dashed lines indicate calculated positions of band gaps in the initial (vertical dash-dotted lines) and final density of states (oblique dashed lines).

curve. It is obvious that none of the models shown in Fig. 11 is able to account for the strong leading peak, which had been assigned to emission from a surface resonance.

\section{B. Emission normal to the (110) face}

Figure 12 shows the calculated band structure together with the one-dimensional density of states along the $\Gamma N$ symmetry line, which corresponds to the (110) crystal face. This part of the tungsten band structure exhibits the interesting feature of two band gaps, one above and one below the Fermi level. These band gaps will be used extensively in the discussion of the experimental data.

The upper gap extends from 6 to about $11 \mathrm{eV}$ above the Fermi level. Within the gap no final states are available for bulk optical excitation, no matter whether direct or nondirect excitation mechanisms are assumed. In other words, no Bloch states are available in the gap to propagate electron wave packets from the bulk to the sur- face with the appropriate $k$ vector for normal emission. On the basis of these arguments photoelectrons emitted in the corresponding energy range are assigned to surface photoemission.

Experimental photoelectron energy-distribution spectra measured normal to the (110) face of tungsten are shown in Fig. 13 for photon energies between 7.7 and $11.7 \mathrm{eV}$. The specta are normalized relative to each other using yield values measured over the total half sphere (as in Fig. 6), and the vertical displacement is such that the excitation energy can be read from the right-hand scale. The bottom scale refers to initial-state energy. The two oblique dashed lines mark the top and the bottom of the conduction band gap, so emission observed between these two lines is assigned to a surface effect. As outlined in Sec. II $\mathrm{C}$, no final-state modulation is expected for structure due to surface emission. Indeed all structure is found to originate from constant initial states, as indicated by the vertical lines marked $A, B$, and $C$. Within the two dashed oblique lines the height of the peaks is also fairly constant, in agreement with the requirements for surface emission, taking into account the limitations in definition of the peak height due to the fact that the curves are not normalized to the true narrow-angle yield. Structure $A$ exhibits a pronounced increase in amplitude as $i_{\imath}^{t}$ crosses the upper limit of the band gap. This is shown in more detail in Fig. 14. Here the peak height of structure $A$ is plotted as a function of excitation photon energy. It is found

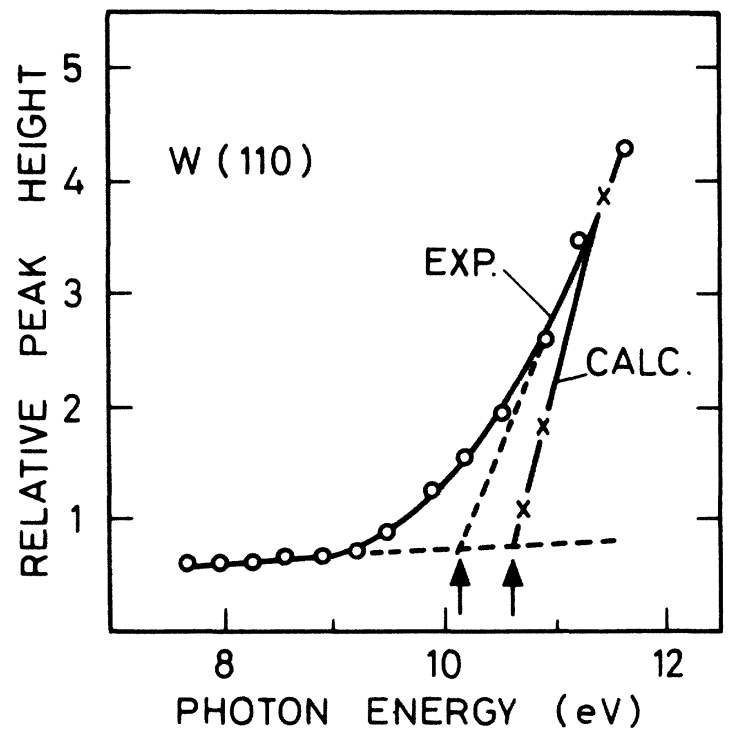

FIG. 14. Relative height of the leading peak in the spectra taken normal to the (110) face (peak $A$ in Fig. 13) as a function of photon energy. Measured values, $\odot$; calculated peak height, $>$. 
nearly constant up to about $9.5 \mathrm{eV}$. The sharp increase for higher photon energies allows an extrapolation to an onset energy for direct transitions of $10.1 \mathrm{eV}$. A theoretical calculation has been performed using the direct-transition model in a $\pm 6^{\circ}$ emission cone normal to (110) surface. ${ }^{38}$ The relative peak size derived from this calculation is also plotted in Fig. 14. A similar sharp increase is found, extrapolating to a threshold energy of $10.6 \mathrm{eV}$. The difference of $0.5 \mathrm{eV}$ between the calculated and observed onset may be due either to the inaccuracy of the calculated band structure, or to a few degrees of surface misalignment, or both. It should be noted that the threshold in the theoretical curve is found slightly lower than the upper limit of the band gap in Fig. 12. This is due to the fact that the density-ofstates curve was calculated strictly one-dimensional, while the direct excitation model includes states which are slightly off the symmetry line but still within the emission cone. The slope of the bands away from the symmetry line causes the effective band gap to decrease in the more realistic direct-excitation calculation.

Whereas the conduction band gap turned out to be helpful in supporting the interpretation of spectra in terms of surface emission, the valence band gap will be used to support the assumption that elastic scattering may be neglected in the present analysis. In Fig. 13, a pronounced scarcity of electrons is found in the energy range -2.5 to about $-5 \mathrm{eV}$. It is assumed that this is related to the band gap in the initial density of states shown in Fig. 12. The very small emission in this energy region allows to set an upper limit to contributions due to umklapp processes, i. e., electrons that have taken up one or more additional reciprocal lattice vectors after the photoexcitation process, or, in other words, to the contribution of higher order plane waves at the surface. The total density of states of tungsten integrated over the entire Brillouin zone, as presented in Ref. 38, shows two large peaks in the above energy range, located at 3 and $4.5 \mathrm{eV}$ below the Fermi level. In the photon energy range 8-12 eV, numerous optical transitions are allowed all over the Brillouin zone, as indicated by the value of the imaginary part of the dielectric constant. ${ }^{38}$ Yet the photoelectron spectra do not show significant contributions in the region of the initial state band gap along the [110] symmetry line. This observation is the main experimental justification for the assumption that elastically scattered electrons may be neglected in the interpretation of the present data, i.e., that the spectra may be related to electronic properties along a single symmetry line in the Brillouin zone, at least for the $[100]$ and the [110] direction. For those two symmetry lines the reciprocal lattice vectors are fully contained in the Brillouin zone. Contributions of umklapp electrons emitted with small or vanishing wave-vector components parallel to the surface will therefore originate from areas on or close to the respective symmetry line in the Brillouin zone. This is, however, not the case for emission normal to the (111) face. Here electrons emitted following a first-order umklapp process involving a [110] reciprocal-lattice vector will originate from the $P H$ line, which is colinear with the $\Gamma P$ line. The amount of this contribution to the spectra measured normal to the (111) face cannot be estimated in the framework of the present paper.

The region of low electron emission in the spectra taken normal to the (110) face does not quite coincide with the calculated position of the band gap. In Fig. 13 the two vertical dash-dotted lines mark the calculated gap. In the experimental curves the gap appears to be shifted by nearly 1 eV toward higher energies. Such a large discrepancy cannot be attributed to sample misalignment or inaccuracy of the calculation, especially in view of the good agreement found for the direct transition calculations in the same energy range for the (100) face. The model calculation for the surface density of states described in Ref. 38 quite naturally leads to such a shift by taking into account the narrowing of the $d$ bands near the surface. In Fig. 15 , a comparison is made between the measured spectrum at 10.2-eV photon energy and calculated curves from various models. The experimental result is plotted as a solid curve in the top frame. The solid curve in Fig. 15(b) shows the calculated contribution due to direct optical transitions. The dashed curve in the same frame is the result of the surface-density-of-states (SDOS) calculation. Comparison to the bulk density of states [dashed curve Fig. 15(c)] demonstrates the shift of the band gap due to the narrowing of the $d$-band at the surface. The two curves in Fig. 15(b) are scaled such that their sum, plotted as a dashed line in the top frame, approximately fits the amplitude of the experimental spectrum. It appears that the very crude model used here gives a reasonable description of the experimental result. In this picture the contribution due to surface emission is about $70 \%$ of the total photoemission. The nondirect model, which is shown as a full curve in Fig. 15(c), predicts no photoemission at all in the range where most of the emission is observed.

Photoemission spectra taken normal to the (110) plane of tungsten are shown for higher excitation energies in Fig. 16. ${ }^{52}$ The bottom scale gives the measured kinetic energy of the emitted electrons. A scale referring the electron energies to the Fermi level is drawn along the baseline of the $21.2-\mathrm{eV}$ 


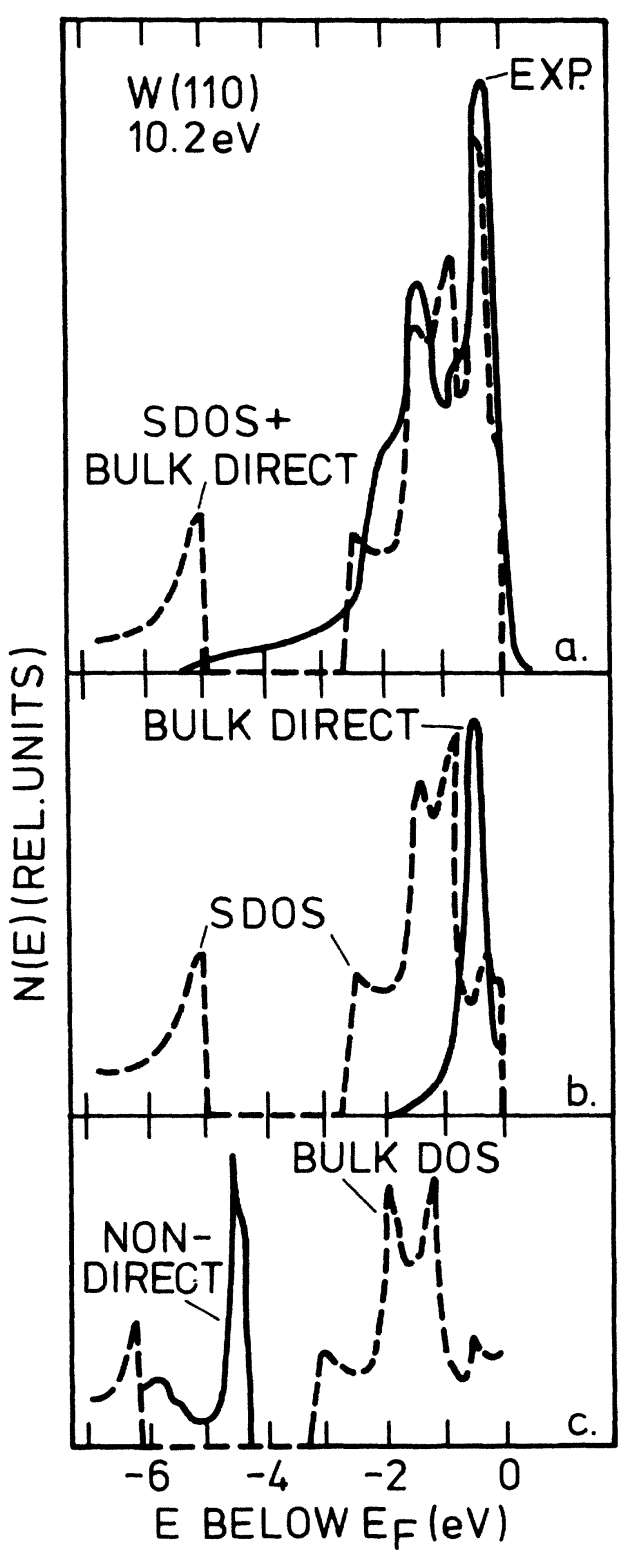

FIG. 15. Photoemission normal to the (110) face of tungsten at 10.2-eV photon energy compared to various calculated models. (a) Solid curve, experimental results; dashed curve, sum of bulk direct transition and surface emission contributions. (b) Solid line, calculated spectra assuming bulk direct transitions (solid line) or surface emission derived from a surface density of states (dashed curve SDOS). (c) Bulk density of states (dashed curve) and calculated spectrum assuming nondirect transitions.

curve. Here a part of the final conduction band density of states shown in Fig. 12 is plotted as a dashed curve. The final-state band gap manifests itself mainly by a general reduction of the number of scattered electrons. This becomes apparent by a comparison to Fig. 10. While for emission from the
(100) face the spectra taken at $21.2 \mathrm{eV}$ are dominated by inelastically scattered electrons, this is not the case for emission normal to the (110) face.

An interesting feature is the prominent peak in the $16.8-\mathrm{eV}$ curve at about $3.4 \mathrm{eV}$ below the Fermi level. This structure is likely to be due to electrons emitted following direct optical excitations, since it is not present at lower or higher photon energies. On the other hand, the initial state of such a transition is right in the gap observed in the spectra for photon energies between 8 and $13 \mathrm{eV}$ (cf. Fig. 13). If the present interpretation is correct, this could be taken as direct evidence for a narrowing of the $d$ bands at the surface, i.e., for a shift of the gap position between the bulk and surface density of states.

\section{Emission normal to the (111) face}

The band structure and the one-dimensional density of states for the $\Gamma P$ symmetry line are shown in Fig. 17. This part of the band structure, which will be used to discrss the results of photoemission normal to the (111) plane, exhibits two rather narrow band gaps, one just below the Fermi level and another one at $9 \mathrm{eV}$ above the Fermi level. Possible contributions due to first-order umklapp electrons arising from the $P H$ line along the face of the Brillouin zone are not included in the calculations of Fig. 17.

The experimental spectra for the photon energy range 7.7-11.7 eV are shown in Fig. 18. A large amount of structure is observed, and the assignment to surface or bulk emission appears to be more involved than for the other two crystal faces discussed above. Structure $A$ and $B$ are likely to be due to surface emission. Their position is independent of photon energy, and their peak size is fairly constant. The dip between $A$ and $B$ accordingly is associated with the lower band gap in Fig. 17. No obvious assignment is at hand for structures $C$ and $D$. Though their initial state position is constant, the peak sizes appear to vary with photon energy. It will be shown that these structures are due to contributions from both surface and volume effects. Structures $E$ and $F$ vary slightly in initial state energy and therefore may be assigned to photoemission following bulk direct transitions.

To obtain a better interpretation of the spectra it is necessary to refer to calculations for the contributions from the bulk direct transition model and the surface emission derived from the surface-density-of-states model. ${ }^{38}$ Figure 19 shows the results of these calculations for $10.2 \mathrm{-eV}$ photon energy. The measured photoelectron spectrum is plotted as a solid line in Fig. 19(a). The calculations predict that the main contribution from bulk direct transitions is a peak at $-3.2 \mathrm{eV}$, shown by a solid line in Fig. 19(b). The dashed line in the same 


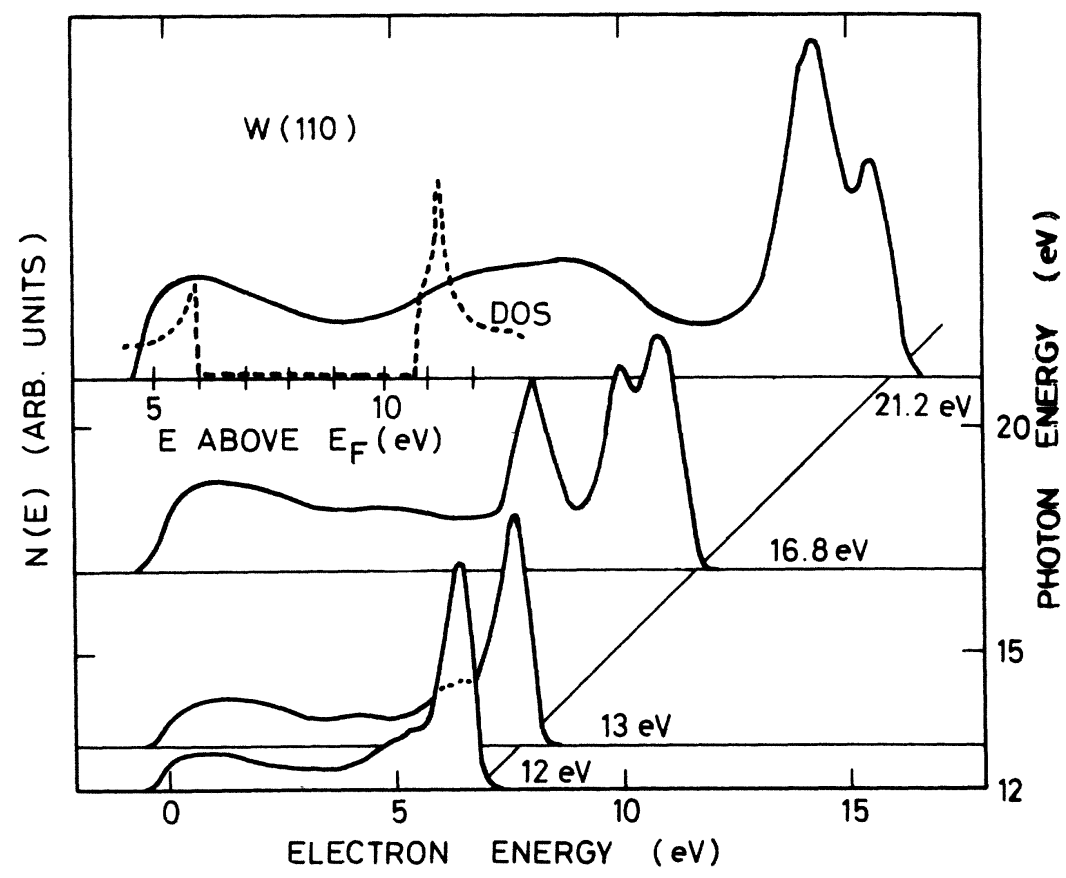

FIG. 16. Energy distribution spectra of photoelectrons emitted normal to the (110) face of tungsten for photon energies between 12 and $21.2 \mathrm{eV}$. The dashed line in the top curve shows a calculated one-dimensional density of states along the $\Gamma N$ symmetry line. frame represents a surface density of states. Comparison to the bulk density of states, shown as a dashed curve in Fig. 19(c), indicates that the main peak is shifted by about $1 \mathrm{eV}$ when going from the bulk to the surface. This shift is introduced by the narrowing of the $d$ bands included in the model. The two curves in Fig. 19(b) are scaled such that their sum, plotted as a dashed line in Fig. 19(a), approximately fits the height of the measured curve. The agreement between the measured spectrum and the sum of the calculated bulk and surface contributions is satisfactory. In this model the contribution of surface emission is $85 \%$ of the total number of electrons photoemitted normal to the (111) face by 10.2-eV photons in the present setup. Spectra for higher excitation energies are presented in Fig. 20.52 The spectrum taken at 13.0$\mathrm{eV}$ photon energy is again compared to the results from the model calculations. The curve marked "bulk direct" has been calculated assuming direct optical transitions and an emission cone of $\pm 6^{\circ}$. The shaded area on top of this curve represents the calculated contribution from surface emission. The agreement of the position of structure in the sum of the calculated curves and the measured spectrum is fair. At this photon energy the model predicts direct transitions to contribute a larger fraction of the total photocurrent than surface emission, a situation quite different from that found at 10.2-eV photon energy. On the other hand, a considerable excess of electrons is found at the lowenergy end of the spectrum, no indication of which was seen in the 10.2-eV spectrum. The low-ener- gy excess is attributed to inelastically scattered electrons. Those become more apparent at higher excitation energies and form the dominating part of the emitted electrons at $21.2-\mathrm{eV}$ photon energy. It is expected that the inelastically scattered electrons reflect the density of final states. For comparison therefore the dashed line marked DOS shows a part of the one-dimensional state density along the $\Gamma P$ line. The calculated band gap at $9 \mathrm{eV}$ above the Fermi level is not well resolved in the measured spectra. This might be due to the finite acceptance angle of the analyzer. The calculated spectrum for bulk direct transitions at $12 \mathrm{eV}$ includes the $\pm 6^{\circ}$ acceptance cone and shows in fact emission with final states right in the gap. On the other hand, umklapp electrons may partly fill up the expected gap. The $P H$ line in the Brillouin zone offers states right through this gap. As discussed above, electrons with final states along this line may be emitted normal to the (111) surface following a first-order umklapp process involving the [110] reciprocal-lattice vector.

\section{DISCUSSION AND CONCLUSIONS}

It is difficult to relate the present results of directional photoemission measurements to any previous photoelectric measurements on tungsten. This is due to the fact that the shape of the photoelectron energy distribution spectra depends in a dramatic way on the acceptance angle of the analyzer, as shown by theoretical calculations based on the direct-transitions model. ${ }^{38}$ Wide-angle measurements are reported by Zeisse ${ }^{55}$ for polycrystalline 


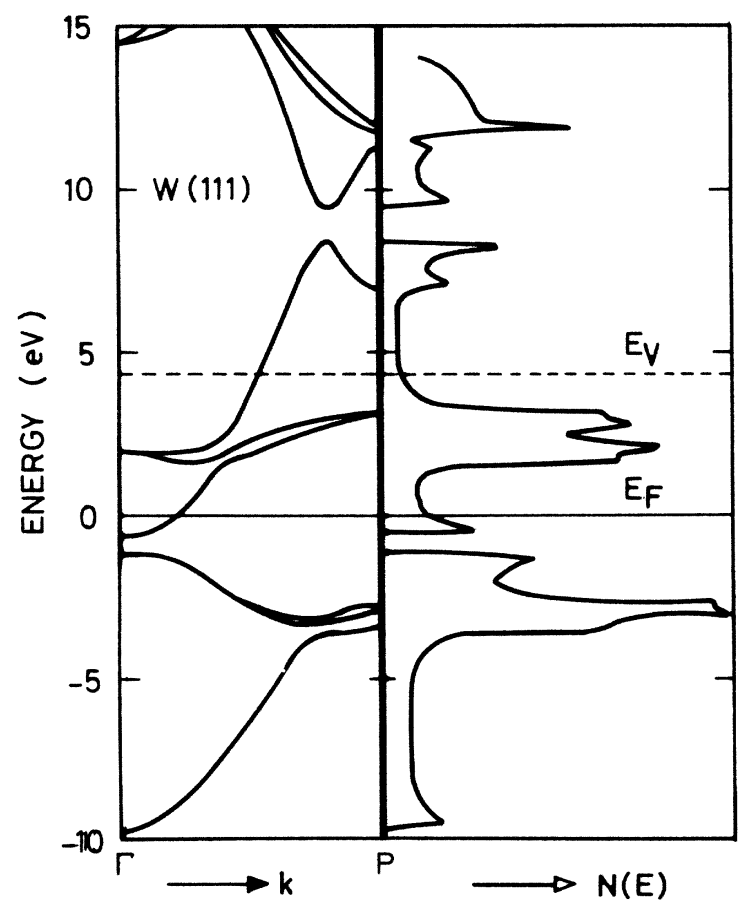

FIG. 17. Calculated band structure of tungsten along the $\Gamma$ P symmetry line (left-hand side) and one-dimensional density of states (right-hand side).

foils and by Baker and Eastman ${ }^{56}$ for single crystals. In the experiments of Waclawski and Plummer $^{44}$ the acceptance angle was limited to $0.2 \mathrm{sr}$, corresponding to about $30^{\circ}$ full cone angle. Though the latter measurements were performed on polycrystalline foils, the strong peak assigned to emission from a surface resonance on the (100) face (peak $A$ in Fig. 6) was observed, indicating some preferential orientation in the foil.

It is interesting to compare the present results to field emission experiments. A set of narrowangle field-emission energy-distribution spectra from tungsten has been reported by Plummer and Bell ${ }^{57}$ for several low-index crystal faces. When compared to the present results at $10.2 \mathrm{-eV}$ photon energy $^{58}$ a very convincing agreement is obtained for all three faces in the region of overlap. This is somewhat surprising in view of the different emission mechanisms acting in the two experiments. However, band structure effects have been considered in field emission theory ${ }^{59,60}$ to influence the energy distribution curves, and it is likely that the surface density of states plays a similar role as suggested here for the photoemission process.

The present interpretation of the photoelectron spectra was based on two assumptions, namely, conservation of the parallel wave vector during emission and negligible elastic scattering. To what extent can these assumptions be justified from the experimental data? Some justification has been given for neglecting elastic scattering. A band gap in the initial surface density of states is reproduced in the observed spectra taken normal to the (110) face [cf. Figs. 13 and $15(\mathrm{a})$ ]. The fact that regions of high densities of initial states in other parts of the Brillouin zone do not contribute significantly in this region of the measured spectra supports the view that elastic processes, including phonon scattering and umklapp processes, may be neglected. Based on this assumption regarding the excitation process, the assumption concerning the emission may also be justified. A first observation is that the spectra from the different faces look very different (cf. Figs. 6, 13, and 18), giving substance to the idea that the spectra carry information from different parts of the Brillouin zone. A closer look at the curves relates parts of the structure to features along single symmetry lines. For example, the inelastically scattered electrons emitted from the (100) face clearly reproduce a band gap in the final states along the $\Gamma H$ symmetry line (see Fig. 10), which is not observed in the emission from other faces. Another example is the structure assigned to direct optical transitions between the

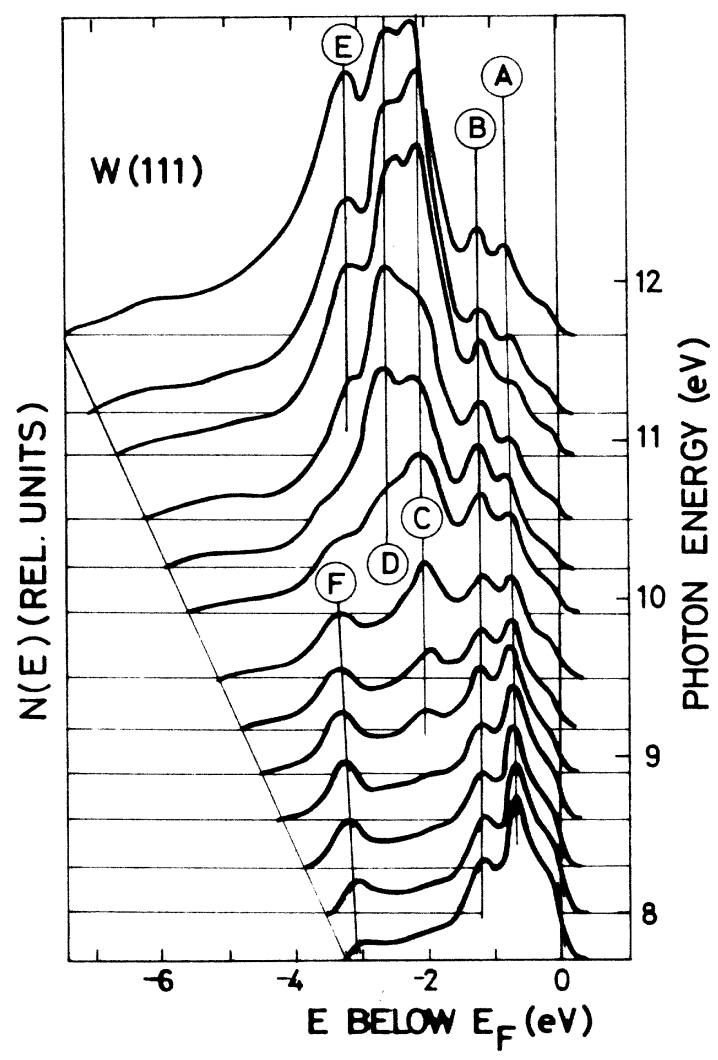

FIG. 18. Experimental energy-distribution spectra of photoelectrons emitted normal to the (111) face of tungsten for photon energies between 7.7 and $11.7 \mathrm{eV}$. 


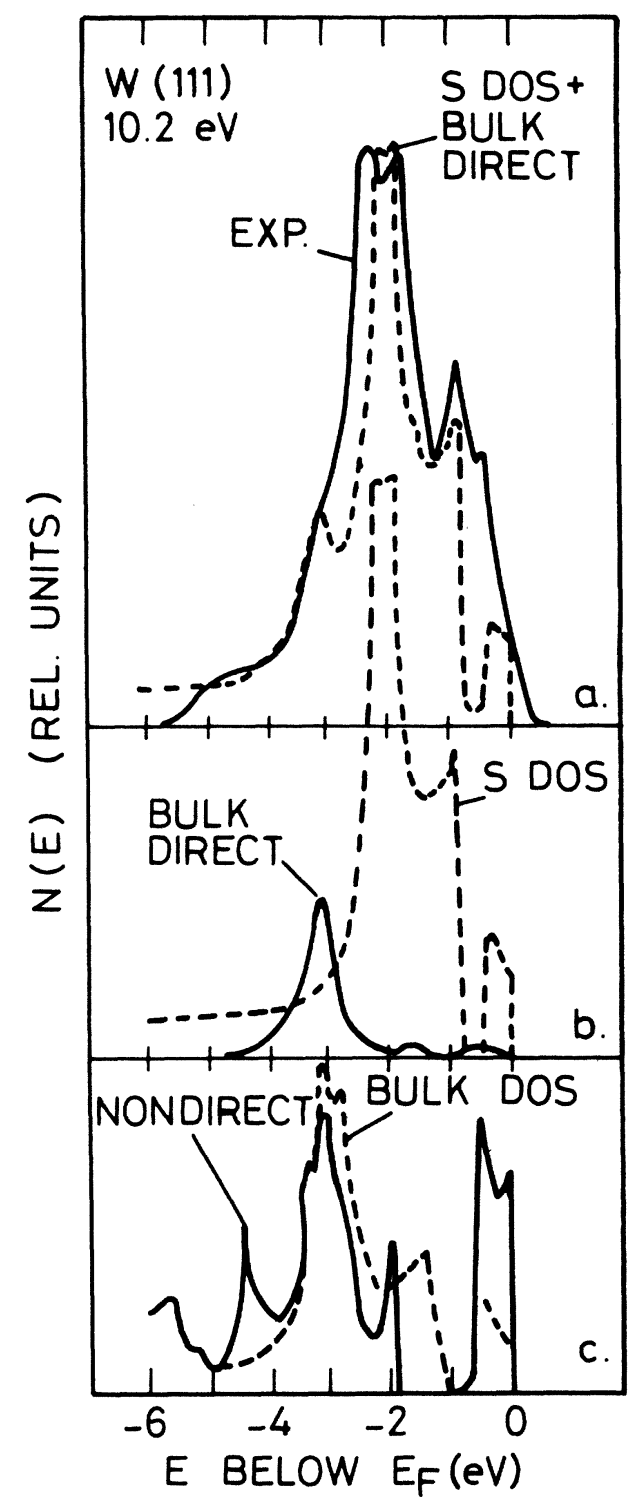

FIG. 19. Photoemission normal to the (111) face of tungsten at 10.2-eV photon energy compared to various calculated models. (a) Solid curve, experimental results; dashed curve, sum of bulk direct transition and surface emission contributions. (b) Solid line, calculated spectra assuming bulk direct transitions (solid line) or surface emission derived from a surface density of states (dashed curve SDOS). (c) Bulk density of states (dashed curve) and calculated spectrum assuming nondirect transitions (solid curve).

bands along the same symmetry line (peak $C$ in Fig. 6; see also Fig. 8). It appears therefore that in the present work the measured spectra may be related primarily to one-dimensional electronic properties along the symmetry line corresponding to the crystal face under observation.
In this aspect, other conclusions may be drawn from the measurements and their analysis. The narrow-angle photoemission spectra permit the emitted electrons to be divided phenomenologically into three separate groups: those electrons which have been excited by direct interband transitions in the bulk and have left the solid unscattered; those electrons which have been inelastically scattered; and finally, those electrons whose emission is specifically from the outermost surface layer. The measured spectrum may thus be regarded as the sum of these three contributions. In the following a few examples which underline the above contributions will be presented and their interpretation will be summarized briefly.

A structure typical of electron emission after direct bulk excitation is peak $C$ in Fig. 6, which shows the movement in initial state energy characteristic of direct processes. It is shown in Fig. 8 that a calculation of the energy density of the joint density of states provides a good description of this type of emission over a considerable range of photon energies. Even the relative peak height for various photon energies can be approximated using this theoretical model (see Fig. 14).

The most striking example for the contribution due to inelastically scattered electrons is, as mentioned above, the emission normal to the (100) face for excitation energies above $16 \mathrm{eV}$ (Fig. 10), where a band gap in the final density of states is reproduced in the spectra. In the present picture

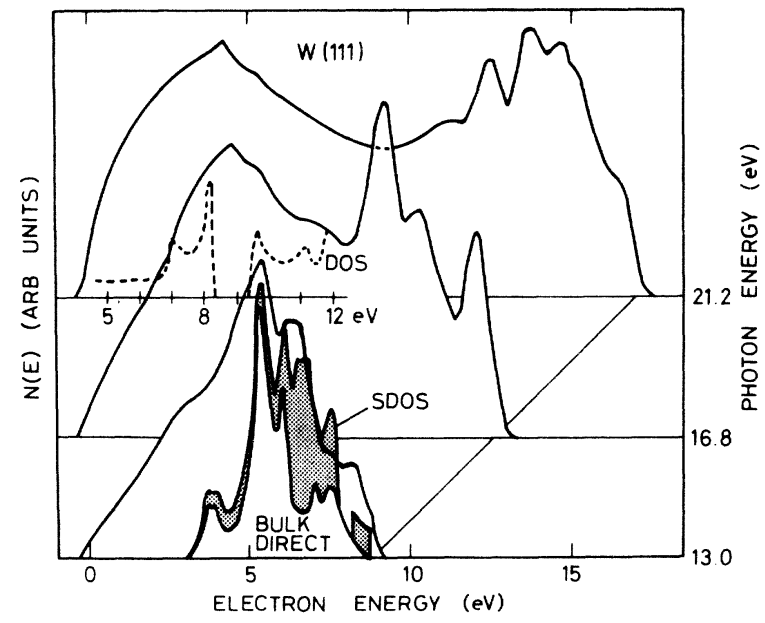

FIG. 20. Energy-distribution spectra of photoelectrons emitted normal to the (111) face of tungsten for $13-, 16.8-$, and 21.2-eV photon energy. The dashed curve shows a calculated one-dimensional density of states along the $\Gamma P$ symmetry line. For $13.0-\mathrm{eV}$ excitation energy calculated contributions are shown due to direct excitation in the bulk (bulk direct) and surface emission derived from a surface density of states (shaded area, SDOS). 
the low-energy electrons are due to electrons excited all over the Brillouin zone that have suffered inelastic scattering events such that the final state happened to be on the symmetry line under observation. No attempt was made in the present work to describe this contribution in a quantitative way..$^{39-41}$

Most of the emission normal to the (110) face has been attributed to a surface effect due to the lack of suitable final states in the band structure (Fig. 12). An attempt to describe the surface emission using the one-dimensional bulk density of states failed, and evidence was found for an appreciable narrowing of the $d$ bands at the surface. This is apparent from a comparison of the calculated band gap for the $\Gamma N$ symmetry line (vertical dash-dotted lines in Fig. 13) and the observed gap, which is shifted to higher energies by nearly $1 \mathrm{eV}$. Note that a high degree of confidence in the band structure calculation for the bulk was gained from the agreement obtained for direct transitions and scattered electrons. A model was therefore developed that takes account of the narrowing of the $d$ bands near the surface due to the reduced number of neighbor atoms. A surface density of states derived from this model provides a good description of the emission attributed to the surface effect [cf. Figs. 11(b) and $15(\mathrm{~b})$ ].

For photon energies around $10 \mathrm{eV}$ the sum of the bulk joint density of states and the surface density of states, both scaled to appropriate amplitudes, gives a fair description of the experimental spectra [see Figs. 19(a) and 15(a)]. The contribution due to the surface effect in the simple model used here appears to be dominant at these energies, for example $85 \%$ of the total emission from the (111) face at $10.2 \mathrm{eV}$ [Fig. 19(b)] in the present experimental setup with a large incidence angle for the light. For higher excitation energies, the direct-transition contribution increases due to the availability of more final-state bands, and at the same time inelastically scattered electrons are observed on the low-energy side of the spectra (bottom curve in Fig. 20).

In the measurements on tungsten shown here no evidence is observed for a significant contribution due to non-direct processes. Furthermore, no description can be given of the strong emission assigned to a surface resonance (peak $A$ in Fig. 6) within the crude model used in the present work.

\section{ACKNOWLEDGMENTS}

The authors are grateful to Dr. E. A. Trendelenburg and Dr. B. Fitton for continuous advice and support during this work, and Dr. R. F. Willis for close cooperation. We gratefully acknowledge the expert technical assistance of $M$. Adriaens throughout the measurements. Thanks are due to Professor J. Blakely of Cornell University for supplying some of the crystals. Figures 2 and 7 have been reproduced from Ref. 51 with permission of North-Holland Publishing Co., Amsterdam.
${ }^{1}$ K. Mitchell, Proc. R. Soc. A 146, 442 (1934).

${ }^{2}$ R. E. B. Makinson, Proc. R. Soc. A 162, 367 (1937).

${ }^{3}$ L. I. Schiff and L. H. Thomas, Phys. Rev. 47,860 (1935).

${ }^{4}$ H. Thomas, Z. Phys. 147, 395 (1957).

${ }^{5} \mathrm{H}$. Mayer and H. Thomas, Z. Phys. 147, 419 (1957).

${ }^{6} \mathrm{H}$. Thomas, in Basic Problems in Thin Film Physics, edited by R. Niedermayer and H. Mayer (Vandenhoek and Ruprecht, Göttingen, 1966), p. 307.

${ }^{7}$ L. Apker, E. Taft, and J. Dickey, Phys. Rev. $\underline{74}, 1462$ (1948).

${ }^{8}$ E. A. Taft and H. R. Philipp, Phys. Rev. $\underline{115}, 1583$ (1959).

${ }^{9}$ W. E. Spicer and R. E. Simon, Phys. Rev. Lett. $\underline{9}$, 385 (1962).

${ }^{10}$ C. N. Berglund and W. E. Spicer, Phys. Rev. 136, A1030 (1964).

${ }^{11}$ C. N. Berglund and W. E. Spicer, Phys. Rev. 136, A1044 (1964).

${ }^{12}$ E. O. Kane, Phys. Rev. $\underline{175}, 1039$ (1968).

${ }^{13}$ I. Adawi, Phys. Rev. 134, A788 (1964).

${ }^{14} \mathrm{D}$. Grant, thesis (The Pennsylvania State University, 1971) (unpublished).

${ }^{15}$ J. G. Endriz, Phys. Rev. B $\underline{7}, 3464$ (1973).

${ }^{16}$ J. G. Endriz and W. E. Spicer, Phys. Rev. Lett. 27, 570 (1971).

${ }^{17}$ J. G. Endriz and W. E. Spicer, Phys. Rev. B $\underline{4}, 4159$ (1971).
${ }^{18}$ G. D. Mahan, Phys. Rev. Lett. 24, 1068 (1970).

${ }^{19}$ G. D. Mahan, Phys. Rev. B 2, 4334 (1970).

${ }^{20}$ N. W. Ashcroft and W. L. Schaich, in Electronic Density of States, edited by L. H. Bennett, Natl. Bur. Stds. Publ. No. 323 (U. S. GPO, Washington, D.C., 1971), p. 129.

${ }^{21}$ W. L. Schaich and N. W. Asheroft, Solid State Commun. 8,1959 (1970).

${ }^{22}$ L. Sutton, Phys. Rev. Lett. 24, 386 (1970).

${ }^{23}$ W. L. Schaich and N. W. Ashcroft, Phys. Rev. B 3 , 2452 (1971).

${ }^{24}$ N. V. Smith, CRC Crit. Rev. Solid State Sci. 2,45 (1971).

${ }^{25}$ D. E. Eastman, in Electron Spectroscopy, edited by

D. Shirley (North-Holland, Amsterdam, 1972), p. 487.

${ }^{26} \mathrm{~N}$. E. Christensen and B. O. Seraphin, Phys. Rev. B 4, 3321 (1971).

${ }^{27}$ N. V. Smith, Phys. Rev. B $\underline{5}, 1192$ (1972).

${ }^{28}$ N. E. Christensen, Phys. Status Solidi B $\underline{54}, 551$ (1972).

${ }^{29}$ N. E. Christensen, Phys. Status Solidi B $\underline{55}, 117$ (1973).

${ }^{30}$ G. W. Gobeli, F. G. Allen, and E. O. Kane, Phys. Rev. Lett. 12, 94 (1964).

${ }^{31}$ E. O. Kane, Phys. Rev. Lett. 12, 97 (1964).

${ }^{32} \mathrm{~J}$. Burns, U. S. Army Electronics Command Night Vision Laboratory Technical Report 1969 (unpublished).

${ }^{33} \mathrm{~F}$. Wooten. T. Huen, and H. V. Winsor, Phys. Lett. 
A 36,351 (1971).

${ }^{34}$ U. Gerhardt and E. Dietz, Phys. Rev. Lett. 26, 1477 (1971).

${ }^{35}$ T. Gustafsson, P. O. Nilsson, and L. Walldén, Phys. Lett. A 37, 121 (1971).

${ }^{36}$ R. Y. Koyama and L. R. Hughey, Phys. Rev. Lett. 29, 1518 (1972).

${ }^{37} \mathrm{~A}$ preliminary account of the present work was given in B. Feuerbacher and B. Fitton, Phys. Rev. Lett. 30, 923 (1973).

${ }^{38}$ N. E. Christensen and B. Feuerbacher, preceding paper, Phys. Rev. B 10, 2349 (1974).

${ }^{39}$ E. O. Kane, Phys. Rev. 159, 624 (1967).

${ }^{40} \mathrm{P}$. O. Nilsson and I. Lindau, in Band Structure Spectroscopy of Metal s and Alloys, edited by D. J. Fabian and L. M. Watson (Academic, New York, 1973).

${ }^{41}$ R. F. Willis, B. Fitton, and G. S. Painter, Phys. Rev. B 9, 1926 (1974).

${ }^{42}$ W. E. Spicer, Phys. Rev. Lett. 11, 243 (1963).

${ }^{43}$ W. E. Spicer, Phys. Rev. 154,385 (1967).

${ }^{44}$ B. J. Waclawski and E. W. Plummer, Phys. Rev. Lett. 29, 783 (1972).

${ }^{45}$ E. W. Plummer and J. W. Gadzuk, Phys. Rev. Lett. 25, 1493 (1970).

${ }^{46} \mathrm{~B}$. Feuerbacher and B. Fitton, Phys. Rev. Lett. 29,
786 (1972).

${ }^{47}$ F. Forstmann and V. Heine, Phys. Rev. Lett. $\underline{24}$, 1419 (1970).

${ }^{48}$ F. Forstmann and J. B. Pendry, Z. Phys. 235,75 (1970).

${ }^{49} \mathrm{~J}$. W. Gadzuk, J. Vac. Sci. Technol. 9, 591 (1972).

${ }^{50}$ D. E. Eastman, Phys. Rev. B 3 , 1769 (1971).

${ }^{51}$ B. Feuerbacher and B. Fitton, Surf. Sci. 37, 386 (1973).

${ }^{52}$ Spectra taken at photon energies above $11.7 \mathrm{eV}$ are not normalized.

${ }^{53}$ H. Kanter, Phys. Rev. B $1,522(1970)$.

${ }^{54}$ R. Haydock, V. Heine, M. J. Kelly, and J. B. Pendry, Phys. Rev. Lett. 29, 868 (1972).

${ }^{55}$ C. R. Zeisse, in Electronic Density of States, edited by L. H. Bennett, Natl. Bur. Stds. Spec. Publ. No. 323 (U. S. GPO, Washington, D. C., 1971), p. 199.

${ }^{56}$ J. M. Baker and D. E. Eastman, J. Vac. Sci. Technol. 10, 223 (1973).

${ }^{57}$ E. W. Plummer and A. E. Bell, J. Vac. Sci. Technol. 9, 583 (1972).

${ }^{58} \overline{\mathrm{E}}$. W. Plummer (private communication).

${ }^{59}$ J. W. Gadzuk, Phys. Rev. 182, 416 (1969).

${ }^{60}$ D. Nagy and P. H. Cutler, Phys. Rev. 186, 651 (1969). 


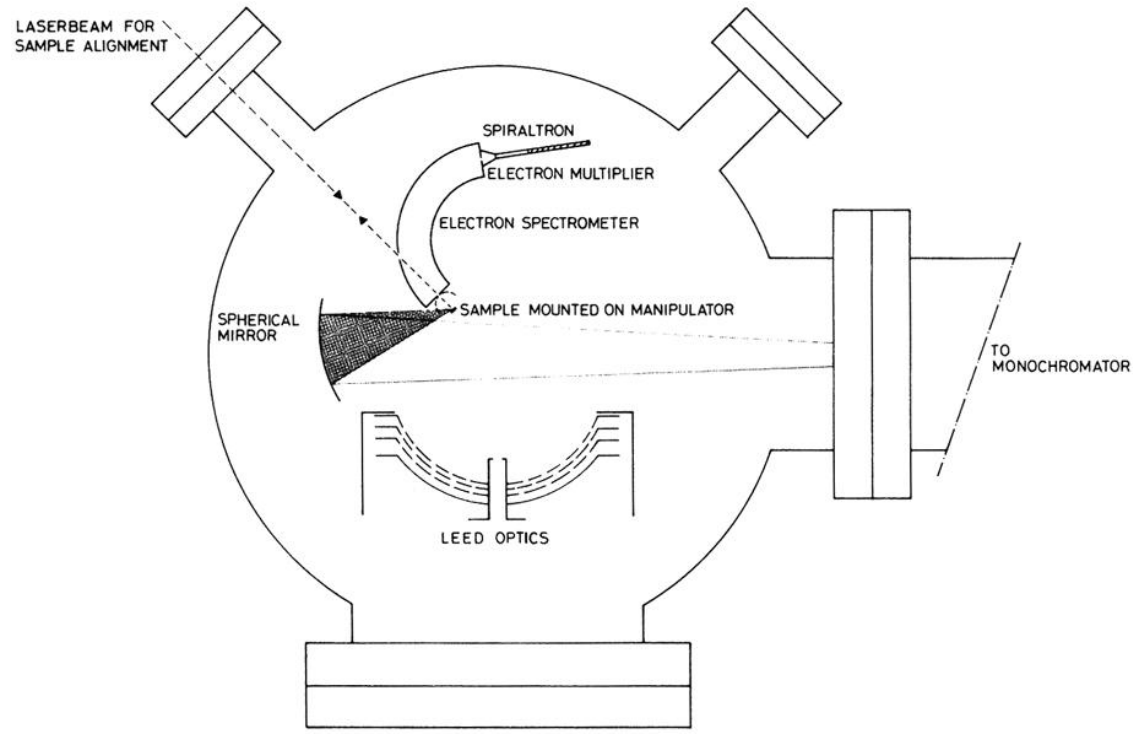

FIG. 2. Schematic layout of the experimental chamber (from Ref. 51). 


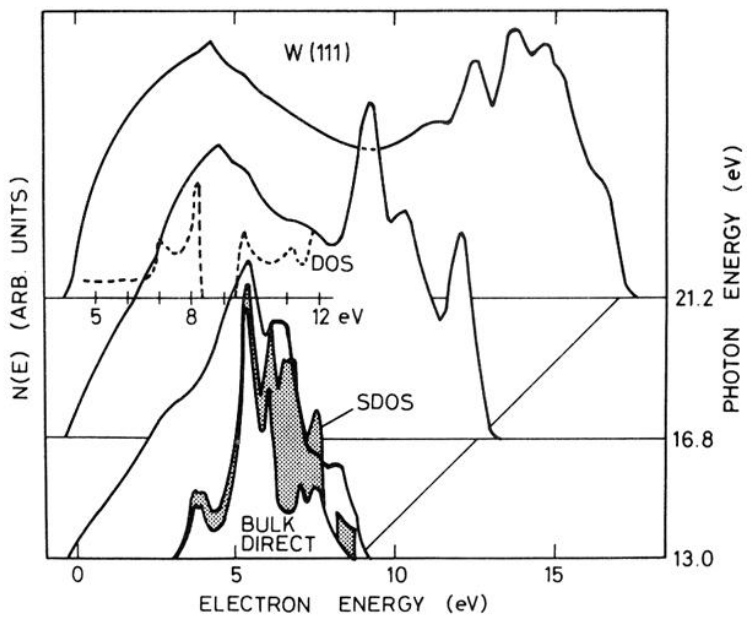

FIG. 20. Energy-distribution spectra of photoelectrons emitted normal to the (111) face of tungsten for 13-, 16.8-, and 21.2-eV photon energy. The dashed curve shows a calculated one-dimensional density of states along the $\Gamma P$ symmetry line. For $13.0-\mathrm{eV}$ excitation energy calculated contributions are shown due to direct excitation in the bulk (bulk direct) and surface emission derived from a surface density of states (shaded area, SDOS). 